\title{
$B$-decay anomalies and scalar leptoquarks in unified Pati-Salam models from noncommutative geometry
}

\author{
Ufuk Aydemir, ${ }^{a, 1}$ Djordje Minic, $^{b}$ Chen Sun ${ }^{c, d, e}$ and Tatsu Takeuchi ${ }^{b}$ \\ ${ }^{a}$ School of Physics, Huazhong University of Science and Technology, \\ Wuhan, Hubei 430074, P.R. China \\ ${ }^{b}$ Center for Neutrino Physics, Department of Physics, Virginia Tech, \\ Blacksburg, VA 24061 U.S.A. \\ ${ }^{c}$ CAS Key Laboratory of Theoretical Physics, Institute of Theoretical Physics, \\ Chinese Academy of Sciences, \\ Beijing 100190, P.R. China \\ ${ }^{d}$ Department of Physics, Brown University, \\ Providence, RI, 02912, U.S.A. \\ ${ }^{e}$ Department of Physics and Astronomy, Dartmouth College, \\ Hanover, NH 03755, U.S.A. \\ E-mail: uaydemir@hust.edu.cn, dminic@vt.edu, chen.sun@brown.edu, \\ takeuchi@vt.edu
}

ABSTRACT: Motivated by possible scalar-leptoquark explanations of the recently reported $B$-decay anomalies, we investigate whether the required leptoquarks can be accommodated within models based on noncommutative geometry (NCG). The models considered have the gauge structure of Pati-Salam models, $\mathrm{SU}(4) \times \mathrm{SU}(2)_{L} \times \mathrm{SU}(2)_{R}$, with gauge coupling unification at a single scale. In one of the models, we find a unique scalar leptoquark with quantum numbers $\left(3,1,-\frac{1}{3}\right)_{321}$, originating from a complex multiplet $(6,1,1)_{422}$, which can potentially explain the $B$-decay anomalies if its mass is on the order of a few $\mathrm{TeV}$. The unification of couplings can be realized with the inclusion of a single step of intermediate symmetry breaking. The scalar leptoquark under consideration does not contribute to proton decay due to the absence of diquark couplings, as dictated by the underlying noncommutative geometry.

Keywords: Beyond Standard Model, Gauge Symmetry, GUT, Non-Commutative Geometry

ARXIV EPRINT: 1804.05844

\footnotetext{
${ }^{1}$ Corresponding author.
} 


\section{Contents}

1 Introduction 1

$2 \quad B$-decay anomalies $\quad 3$

3 Scalar leptoquarks $\quad 5$

4 Scalar leptoquarks in unified Pati-Salam models from NCG $\quad 7$

$\begin{array}{lll}4.1 & \text { NCG model construction } & 7\end{array}$

4.2 The three models $\quad 9$

$\begin{array}{ll}4.3 \text { Couplings between fermions and scalars } & 10\end{array}$

$\begin{array}{lll}\text { 4.3.1 } & \text { Model A } & 12\end{array}$

$\begin{array}{lll}4.3 .2 & \text { Model B } & 15\end{array}$

$\begin{array}{lll}\text { 4.3.3 } & \text { Model C } & 17\end{array}$

$\begin{array}{llr}5 & \text { Gauge coupling unification } & 18\end{array}$

$\begin{array}{lll}5.1 & \text { 1-loop renormalization group running } & 19\end{array}$

5.2 Does the leptoquark help coupling unification? 20

$\begin{array}{lll}5.3 & \text { Unification with a single intermediate scale } 25\end{array}$

6 Discussion and conclusions $\quad 27$

6.1 Discussion 27

6.2 Light colored scalars and proton decay 28

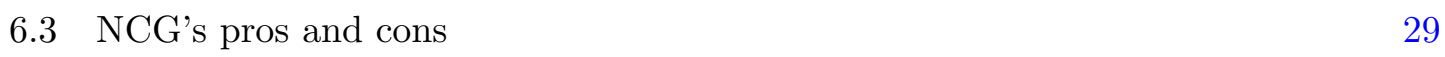

$\begin{array}{ll}\text { A Leptoquarks } & 30\end{array}$

\section{Introduction}

In papers [1] and [2] we have studied the phenomenology of models proposed by Chamseddine, Connes, and van Suijlekom in refs. [3, 4], which are based on Connes' noncommutative geometry (NCG) [5, 6]. The models are characterized by a Pati-Salam (PS) gauge structure $G_{422}=\mathrm{SU}(4) \times \mathrm{SU}(2)_{L} \times \mathrm{SU}(2)_{R}$ with the requirement that the gauge couplings unify at a single scale, and a scalar sector whose content and couplings are fixed by the NCG of the models. The fermion content is the same as the Standard Model (SM) plus the right-handed neutrinos of each generation.

The unification of gauge couplings is a distinguishing requirement of NCG based versions of the SM [7, 8], as well as its $G_{422}$ extensions discussed in this paper [3, 4]. It is due to the underlying "spectral action" having only one overall coupling, despite the fact that the gauge groups do not unify into a single simple Lie group, that is, the model is 
not a Grand Unified Theory (GUT). Note that coupling unification is not a requirement of canonical non-GUT $G_{422}$ models found in the literature [9-13].

Another feature of the NCG-PS framework is its restricted scalar content compared to the canonical Pati-Salam and GUT frameworks, which is appealing in terms of the predictability of the NCG framework. The scalar content of the three NCG-PS models proposed in refs. [3, 4] is listed in table 1 and their full SM decomposition can be inferred from table 2. As shown in refs. [3, 4], the scalar sector is restricted due to the geometric feature of NCG as outlined in section 4.1. In essence, as the Higgs field is the gauge field in the discrete direction of NCG space, requiring gauge invariance in the total NCG space puts a stronger restriction on the Higgs sector than requiring it only in Minkowski space, hence reducing the number of free parameters and possible combination of terms in the Higgs potential. ${ }^{1}$

The RG running in the NCG-PS framework is treated adopting the usual effective field theory approach. The difference between the canonical and NCG based Pati-Salam models is in the restricted scalar content of the NCG-PS formalism. The underlying noncommutative geometry, which is interpreted as the classical background, is assumed to be relevant at the UV. The SM and PS structures are interpreted as emergent from the corresponding underlying noncommutative geometry. ${ }^{2}$

In ref. [1], we investigated whether a $2 \mathrm{TeV} W_{R}$ boson [14-19] could be accommodated within the NCG models considered, and in ref. [2] whether a $750 \mathrm{GeV}$ diphoton resonance $[20,21]$ could be accommodated. Due to constraints imposed on the models by the underlying NCG, no freedom exists to adjust the models' particle content, except via decoupling those that exist from the renormalization group (RG) running of the gauge coupling constants by rendering them heavy through symmetry breaking. Consequently, though it is not difficult to find particles within the models that can be identified with the putative $W_{R}$ or the diphoton resonance, we have found that lowering their masses to $\mathrm{TeV}$ scales while maintaining the coupling unification condition is highly non-trivial. The hints of the $W_{R}$ and diphoton resonance at the LHC have since disappeared [22-24], but our analyses nevertheless underscore the rigidity, and consequently the predictability, of the NCG based models.

In this paper, we continue our investigation and question whether the models could naturally accommodate a $\mathrm{TeV}$-scale scalar leptoquark necessary to explain the $B$-decay anomalies observed at the LHC and elsewhere [25]. The leptoquark of interest is $S_{1}\left(\overline{3}, 1, \frac{1}{3}\right)_{321}$, a colored isoscalar with electromagnetic charge $\frac{1}{3}$. In order to explain the $B$-decay anomalies, its mass must be in the few $\mathrm{TeV}$ range [26-29]. Again, it is not difficult to identify scalars with the required quantum numbers within the NCG models, but the quantum number assignment alone does not necessarily guarantee that the scalars couple to lepton-quark pairs

\footnotetext{
${ }^{1}$ The fermion masses in NCG framework, as in the SM, are input in the NCG framework, which are contained in the generalized Dirac operator that is used to construct the NCG Lagrangian. In both the spectral Standard Model and its Pati-Salam extension, the smallness of the neutrino masses can be addressed in the context of seesaw mechanism $[7,8]$.

${ }^{2}$ It is currently unknown if there are non-local degrees of freedom due to the geometric nature of the framework that may change the RG running in a non-Wilsonian way.
} 
as leptoquarks should do. And again, the challenge is whether coupling unification can be achieved or not. On this question, we recall that in the context of SU(5) GUT, the presence of certain leptoquarks actually helps in unifying the couplings without SUSY [33, 34]. Our study will focus on whether the field corresponding to the $S_{1}$ has a similar effect in NCG models.

Another appealing aspect of the NCG-based Pati-Salam models, pointed out in this paper, is the proton stability. Ordinarily, in the usual Pati-Salam framework the stability of proton is not always guaranteed in the case of $S_{1}$ being light. In the NCG formalism, on the other hand, the proton stability is ensured thanks to the absence of $S_{1}$ 's diquark couplings, as dictated by the underlying noncommutative geometry.

This paper is organized as follows: In section 2 we briefly review the current status of $B$-decay anomalies. In section 3 we list the various leptoquark explanations that have been proposed in the literature, and identify the introduction of a single $S_{1}$ leptoquark as the most attractive solution. Section 4 discusses the scalar content of the NCG models and their Yukawa couplings, and we find a unique field that would serve our purpose as the $S_{1}$ leptoquark. Section 5 presents an analysis on how and whether the coupling unification condition can be maintained when the $S_{1}$ mass is a few TeV. Section 6 concludes with a discussion of how the proton decay is, perhaps surprisingly, not a problem in our context, as well as some concluding comments on the NCG framework and the current status of noncommutativity in quantum gravity and string theory. We end with an appendix in which we review the derivation of the most generic Lagrangian for scalar and vector leptoquark interactions with Standard Model (SM) fermions.

\section{$2 \quad B$-decay anomalies}

During the past few years, several disagreements between experiment and Standard Model (SM) predictions in rare B-decays have been observed by LHCb [35-39], Belle [40-42], and Babar [43, 44]. The decay channels in question are $B^{+} \rightarrow K^{+} \mu^{+} \mu^{-}[35], B^{0} \rightarrow$ $K^{* 0} \mu^{+} \mu^{-}[36,37]$, and $\overline{B^{0}} \rightarrow D^{(*)+} \tau^{-} \bar{\nu}[38-44]$. Expressed in terms of the ratios

$$
R_{K^{(*)}}=\frac{\mathcal{B}\left(B \rightarrow K^{(*)} \mu^{+} \mu^{-}\right)}{\mathcal{B}\left(B \rightarrow K^{(*)} e^{+} e^{-}\right)}
$$

LHCb reports $[35-37]^{3}$

$$
\begin{aligned}
& R_{K}=0.745_{-0.074}^{+0.090} \text { (stat) } \pm 0.036 \text { (syst) for } 1<q^{2}<6 \mathrm{GeV}^{2} / \mathrm{c}^{4} \text {, } \\
& R_{K^{*}}= \begin{cases}0.660_{-0.070}^{+0.110}(\text { stat }) \pm 0.024 \text { (syst) } & \text { for } 0.045<q^{2}<1.1 \mathrm{GeV}^{2} / \mathrm{c}^{4} \\
0.685_{-0.069}^{+0.113} \text { (stat) } \pm 0.047 \text { (syst) } & \text { for } 1.1<q^{2}<6.0 \mathrm{GeV}^{2} / \mathrm{c}^{4}\end{cases}
\end{aligned}
$$

where $q^{2}$ is the invariant mass of the lepton pair in the final state. Here, the upper cut $q_{\text {max }}^{2}=6 \mathrm{GeV}^{2} / \mathrm{c}^{4}$ is imposed to avoid the effects of the $J / \psi$ and higher $c \bar{c}$ resonances,

\footnotetext{
${ }^{3}$ Previous measurements of these ratios by Belle and Babar were consistent with the SM within their larger experimental uncertainties.
} 
while a common lower cut $q_{\text {min }}^{2}$ for both electron and muon final states is imposed to avoid phase space effects. In the case of $R_{K^{*}}$, the lower cut has been taken all the way down to $q_{\min }^{2}=0.045 \mathrm{GeV}^{2} / \mathrm{c}^{4} \approx 4 m_{\mu}^{2}$, but the range from this $q_{\min }^{2}$ to $q_{\max }^{2}=6 \mathrm{GeV}^{2} / \mathrm{c}^{4}$ has been divided into two at $q^{2}=1.1 \mathrm{GeV}^{2} / \mathrm{c}^{4}$ in order to isolate the contribution of the $\phi$ resonance $\left(B^{0} \rightarrow \phi\left(\rightarrow \ell^{+} \ell^{-}\right) K^{* 0}\right)$ into the lower bin. With these bounds on $q^{2}$ in place, hadronic uncertainties have been argued to cancel in these ratios and that the SM predictions of $R_{K}$ and $R_{K^{*}}$ are unity with only $O\left(10^{-4}\right)$ uncertainties [45-49]. However, it has also been argued that both QED [50] and QCD [51] corrections and uncertainties were underestimated in these predictions. Ref. [50] argues that a more realistic set of SM predictions is

$$
\begin{array}{ll}
R_{K}^{\mathrm{SM}}[1,6] & =1.00 \pm 0.01, \\
R_{K^{*}}^{\mathrm{SM}}[1.1,6] & =1.00 \pm 0.01, \\
R_{K^{*}}^{\mathrm{SM}}[0.045,1.1] & =0.906 \pm 0.028,
\end{array}
$$

while ref. [51] lists

$$
\begin{aligned}
& R_{K^{*}}^{\mathrm{SM}}[1.1,6]=1.000 \pm 0.006, \\
& R_{K^{*}}^{\mathrm{SM}}[0.045,1.1]=0.922 \pm 0.022 .
\end{aligned}
$$

Thus, the prediction that the values of $R_{K}^{\mathrm{SM}}[1,6]$ and $R_{K^{*}}^{\mathrm{SM}}[1.1,6]$ are unity appears to be fairly robust, and we find that the experimental values of $R_{K}[1,6]$ and $R_{K^{*}}[1.1,6]$ are both suppressed compared to their SM predictions by $\sim 2.6 \sigma[35-37]$.

For the semileptonic $B$ to $D$ decays, the ratios

$$
R_{D^{(*)}}=\frac{\mathcal{B}\left(B \rightarrow D^{(*)} \tau \nu\right)}{\mathcal{B}\left(B \rightarrow D^{(*)} \ell \nu\right)}, \quad \ell=e \text { or } \mu
$$

have been measured using a variety of techniques by Babar [43, 44], Belle [40-42], and $\mathrm{LHCb}\left(R_{D^{*}}\right.$ only) $[38,39]$. For the denominator of the above ratios, Belle and Babar take the average of $B \rightarrow D^{(*)} e \nu$ and $B \rightarrow D^{(*)} \mu \nu$ branching fractions, while LHCb uses that for $B \rightarrow D^{(*)} \mu \nu$. The world averages as of summer 2017, according to the Heavy Flavor Averaging Group [52], are

$$
\begin{aligned}
& R_{D}=0.407 \pm 0.039(\text { stat }) \pm 0.024(\text { syst }) \\
& R_{D^{*}}=0.304 \pm 0.013(\text { stat }) \pm 0.007 \text { (syst) } .
\end{aligned}
$$

The Standard Model (SM) predictions of these ratios, on the other hand, are [53, 54] ${ }^{4}$

$$
\begin{aligned}
& R_{D}^{\mathrm{SM}}=0.300 \pm 0.008, \\
& R_{D^{*}}^{\mathrm{SM}}=0.252 \pm 0.003,
\end{aligned}
$$

and one sees that the experimental values $R_{D}$ and $R_{D^{*}}$ are in excess of their SM predictions by $2.3 \sigma$ and $3.4 \sigma$, respectively [52].

These experimental results, ${ }^{5}$ taken at face value, challenge lepton flavor universality [57] and could be interpreted as signatures of new physics beyond the SM. Note that for

\footnotetext{
${ }^{4}$ See also the recent analyses in ref. [55] and ref. [56], both of which obtain slightly different values for these ratios.

${ }^{5}$ See also ref. [25] for a recent review.
} 


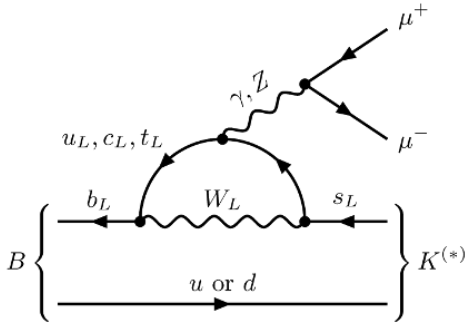

(a)

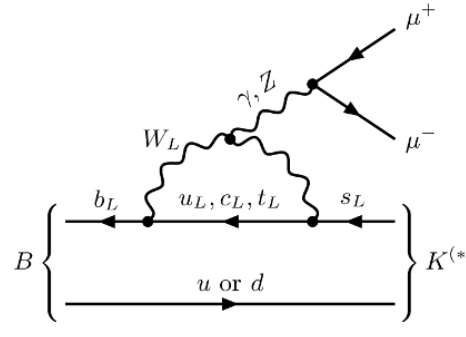

(b)

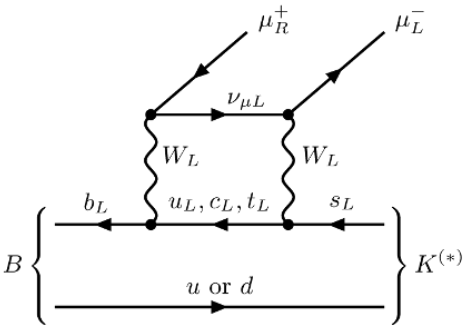

(c)

Figure 1. SM processes that contribute to $B \rightarrow K^{(*)} \mu^{+} \mu^{-}$. The muons in the final states of (a) and (b) do not have definite chirality.

new physics to account for $R_{K^{(*)}}<R_{K^{(*)}}^{\mathrm{SM}}$ one could either suppress the numerator or enhance the denominator, while for $R_{D^{(*)}}>R_{D^{(*)}}^{\mathrm{SM}}$ one could either enhance the numerator or suppress the denominator. However, any new physics involving the electron is generically highly constrained. Therefore, new physics needs to suppress the process $B \rightarrow K^{(*)} \mu^{+} \mu^{-}$, while enhancing the process $B \rightarrow D^{(*)} \tau \nu_{\tau}$.

\section{Scalar leptoquarks}

Unlike several other LHC "anomalies" that have come and gone [22-24] the persistence of the $B$-decay anomalies has garnered a great deal of interest and various explanations involving new physics have been proposed. Here, we focus our attention to those involving scalar leptoquarks. ${ }^{6}$

As reviewed in the appendix, there exist six possible $G_{321}=\mathrm{SU}(3)_{C} \times \mathrm{SU}(2)_{L} \times \mathrm{U}(1)_{Y}$ quantum number assignments to scalar leptoquarks as listed in table 7 . The corresponding six scalar leptoquark fields are labelled $S_{1}, \widetilde{S}_{1}, \bar{S}_{1}, S_{3}, R_{2}$, and $\widetilde{R}_{2}$. Their couplings to the quarks and leptons are shown explicitly in eqs. (A.10) and (A.11) and also summarized in the last column of table 7 .

To explain the $R_{K^{(*)}}$ anomalies one needs to induce $b_{L} \rightarrow s_{L} \mu^{+} \mu^{-}$operators which can interfere destructively with the SM processes shown in figure 1. These operators are denoted in the literature as $[60-62]^{7}$

$$
\begin{aligned}
& \mathcal{O}_{9} \propto\left(\overline{s_{L}} \gamma^{\mu} b_{L}\right)\left(\bar{\mu} \gamma_{\mu} \mu\right)=2\left(\overline{s_{L}} \mu_{R}\right)\left(\overline{\mu_{R}} b_{L}\right)+2\left(\overline{b_{L}^{C}} \mu_{L}\right)\left(\overline{\mu_{L}} s_{L}^{C}\right) \\
& \mathcal{O}_{10} \propto\left(\overline{s_{L}} \gamma^{\mu} b_{L}\right)\left(\bar{\mu} \gamma_{\mu} \gamma_{5} \mu\right)=2\left(\overline{s_{L}} \mu_{R}\right)\left(\overline{\mu_{R}} b_{L}\right)-2\left(\overline{b_{L}^{C}} \mu_{L}\right)\left(\overline{\mu_{L}} s_{L}^{C}\right) .
\end{aligned}
$$

Looking through the leptoquark-quark-lepton couplings listed in eqs. (A.10) and (A.11), we can see that in order to induce these operators at tree-level one needs $S_{3}^{(4 / 3)}$, which induces

\footnotetext{
${ }^{6}$ For a recent general review on leptoquark-related phenomenology, see ref. [58]. The classic reference on leptoquarks is ref. [59]. For completeness, we review the basic theoretical facts from these references in the appendix.

${ }^{7}$ Ref. [60] uses a slightly different numbering scheme in which $\mathcal{O}_{9 / 10}$ are respectively denoted $\mathcal{O}_{8 / 9}$. The normalizations of the operators also differ among various publications so care is necessary when comparing the sizes of the Wilson coefficients.
} 


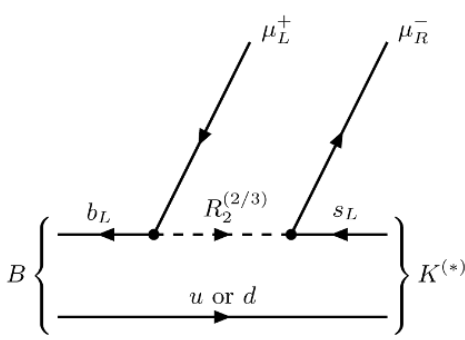

(a)

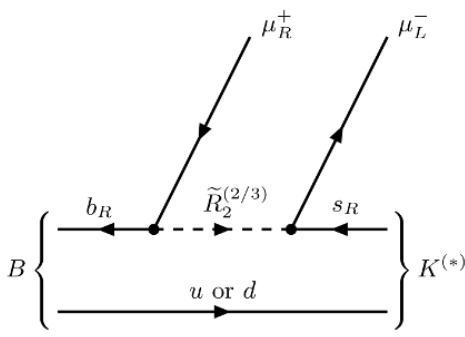

(d)

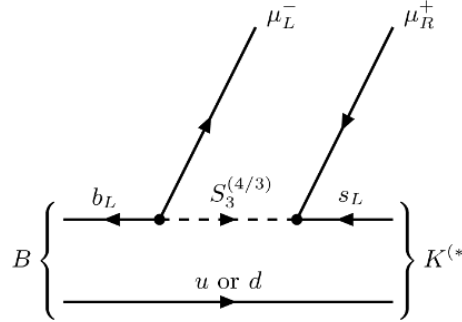

(b)

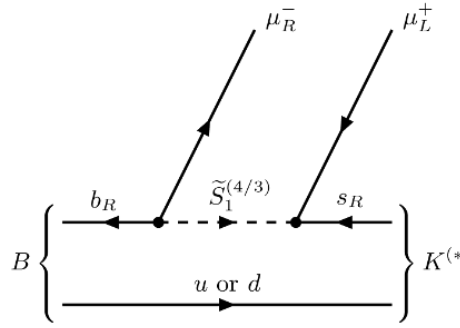

(e)

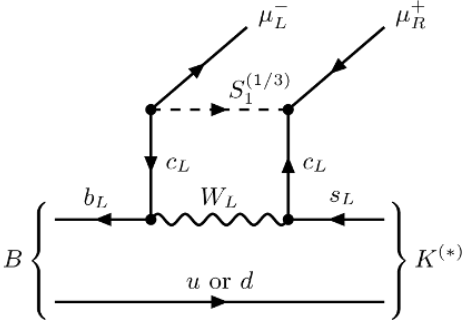

(c)

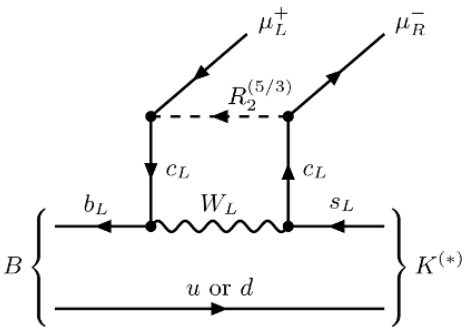

(f)

Figure 2. Possible leptoquark contributions to $B \rightarrow K^{(*)} \mu^{+} \mu^{-}$.

$\mathcal{O}_{9}-\mathcal{O}_{10}[48,63,64]$, or $R_{2}^{(2 / 3)}$, which induces $\mathcal{O}_{9}+\mathcal{O}_{10}[27,48,65]$. See figures 2(a) and $2(\mathrm{~b})$. The wrong chirality operators

$$
\begin{aligned}
& \mathcal{O}_{9}^{\prime} \propto\left(\overline{s_{R}} \gamma^{\mu} b_{R}\right)\left(\bar{\mu} \gamma_{\mu} \mu\right)=2\left(\overline{b_{R}^{C}} \mu_{R}\right)\left(\overline{\mu_{R}} s_{R}^{C}\right)+2\left(\overline{s_{R}} \mu_{L}\right)\left(\overline{\mu_{L}} b_{R}\right), \\
& \mathcal{O}_{10}^{\prime} \propto\left(\overline{s_{R}} \gamma^{\mu} b_{R}\right)\left(\bar{\mu} \gamma_{\mu} \gamma_{5} \mu\right)=2\left(\overline{b_{R}^{C}} \mu_{R}\right)\left(\overline{\mu_{R}} s_{R}^{C}\right)-2\left(\overline{s_{R}} \mu_{L}\right)\left(\overline{\mu_{L}} b_{R}\right),
\end{aligned}
$$

which contribute to $b_{R} \rightarrow s_{R} \mu^{+} \mu^{-}$, are obtained from the exchange of $\widetilde{R}_{2}^{(2 / 3)}$, which induces $\mathcal{O}_{9}^{\prime}-\mathcal{O}_{10}^{\prime}[27,34,48,63,66-68]$, or $\widetilde{S}_{1}^{(4 / 3)}$, which in turn induces $\mathcal{O}_{9}^{\prime}+\mathcal{O}_{10}^{\prime}[48,63]$. See figures 2(d) and 2(e). Global fits that have been performed by various groups, e.g. refs. [48, 49, 69-71], indicate that a suppression of the Wilson coefficient $C_{9}$ (the coefficient of $\mathcal{O}_{9}$ ) compared to its SM value by about $25 \%$ provides the best fit.

For the $R_{D^{(*)}}$ anomalies we need an operator which interferes constructively with the SM process shown in figure $3(\mathrm{a})$.

The required operator is

$$
\left(\overline{c_{L}} \gamma^{\mu} b_{L}\right)\left(\overline{\tau_{L}} \gamma_{\mu} \nu_{\tau L}\right)=\left(\overline{b_{L}^{C}} \gamma^{\mu} c_{L}^{C}\right)\left(\overline{\tau_{L}} \gamma_{\mu} \nu_{\tau L}\right)=2\left(\overline{b_{L}^{C}} \nu_{\tau L}\right)\left(\overline{\tau_{L}} c_{L}^{C}\right)
$$

To induce this at tree-level one needs $S_{1}^{(1 / 3)}[26-32]$ or $S_{3}^{(1 / 3)}$ [30-32] which couple to left-handed quarks and leptons. See figure $3(\mathrm{~b})$. We find the introduction of the $S_{1}^{(1 / 3)}$ leptoquark coupled to left-handed fermions particularly attractive since, as pointed out in ref. [26], in addition to explaining the $R_{D^{*}}$ anomalies at tree level, it can also explain the $R_{K^{(*)}}$ anomalies at the one-loop level, the same level as the SM contributions. ${ }^{8}$ See figure 2(c). Under closer scrutiny, it was suggested in ref. [27] that the $S_{1}^{(1 / 3)}$ model may

\footnotetext{
${ }^{8}$ See refs. [30-32] for possible two-leptoquark solutions that utilize $S_{1}\left(\overline{3}, 1, \frac{1}{3}\right)_{321}$ and $S_{3}\left(\overline{3}, 3, \frac{1}{3}\right)_{321}$.
} 


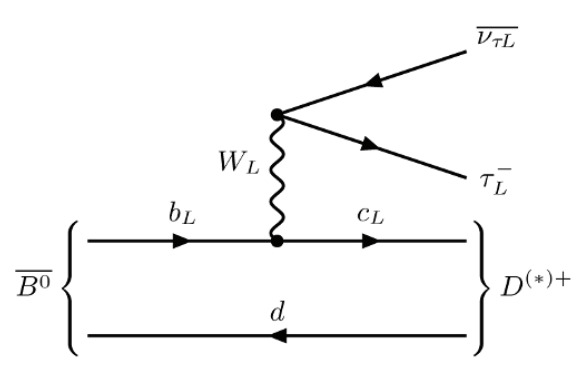

(a)

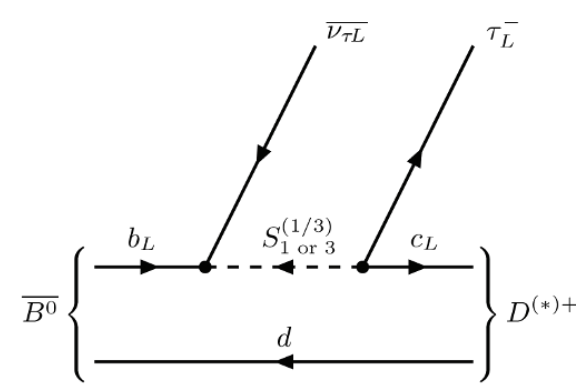

(b)

Figure 3. (a) SM and (b) possible leptoquark contributions to $\bar{B}_{0} \rightarrow D^{(*)+} \tau^{-} \overline{\nu_{\tau L}}$.

not be able to explain $R_{K^{(*)}}$ without coming into conflict with the experimental values of the ratios

$$
R_{D^{(*)}}^{\mu / e}=\frac{\mathcal{B}\left(B \rightarrow D^{(*)} \mu \nu\right)}{\mathcal{B}\left(B \rightarrow D^{(*)} e \nu\right)}
$$

However, ref. [28] argues that this constraint can be circumvented by allowing the $S_{1}^{(1 / 3)}$ mass to be a $\mathrm{TeV}$ or larger, the preferred mass being a few $\mathrm{TeV}$.

Assuming that an $S_{1}^{(1 / 3)}$ leptoquark coupled to left-handed fermions with a mass of a few TeV can simultaneously explain the $R_{K^{(*)}}$ and $R_{D^{(*)}}$ anomalies, we inquire whether such a leptoquark can be accommodated within the NCG models of Chamseddine et al. [3, 4].

\section{Scalar leptoquarks in unified Pati-Salam models from NCG}

In this section, we list the three NCG-based unified Pati-Salam-like models proposed by Chamseddine, Connes, and van Suijlekom in refs. [3, 4], and specify how leptoquarks can be fit into their particle contents. We begin with a brief outline of how NCG models are constructed, in particular, the three models of refs. [3, 4].

\subsection{NCG model construction}

Noncommutative geometry (NCG) generalizes the concepts of spinor bundle and principle bundle, and manages to combine the two concepts into one in a mathematically rigorous fashion [5]. As a result, the Dirac operator has both a continuous part and a discrete part. The continuous part realizes the ordinary Dirac operator, and the discrete part accounts for the representation space of the gauge algebra.

Consequently, in this framework, the most general gauge transformation includes both a transformation along the continuous directions, which is identified with the usual gauge transformation, and that in the discrete direction, which is not explicit in the usual field theoretical formalism. To maintain gauge invariance of the whole action, one needs gauge fields in both directions, which are interpreted as the ordinary gauge fields (continuous direction) and the Higgs fields (discrete direction). 
It is worth noting that if one tries to interpret the space as an extra-dimension model, the metric dimension of the extra dimension is zero, because the extra direction is discrete. Therefore, in contrast to the usual extra-dimension models, no extra effort is required to stabilize the two "sheets." In what follows, we show this more concretely. The continuous gauge fields $A_{\mu}$ transform in the usual way:

$$
\begin{aligned}
A_{\mu}^{\prime} & =u A_{\mu} u^{-1}-\frac{i}{g} u \partial_{\mu} u^{-1} \\
& =A_{\mu}+u\left[A_{\mu}, u^{-1}\right]-\frac{i}{g} u\left[\partial_{\mu}, u^{-1}\right]
\end{aligned}
$$

where $u$ is an element of the gauge group, while the discrete part, the Higgs field $\Phi$, transforms in an analogous fashion with $\partial_{\mu}$ replaced by a matrix commutator $[D, \cdot]$, which represents a "derivative" in the discrete direction: ${ }^{9}$

$$
\Phi^{\prime}=\Phi+u\left[\Phi, u^{-1}\right]-\frac{i}{g} u\left[D, u^{-1}\right] .
$$

Here, the matrix $u$ is still a transformation generated by the gauge algebra, and the matrix $D$ is the discrete part of the Dirac operator. In NCG, the gauge field is defined as as the summation $\sum a[\mathcal{D}, b]$, with $a, b$ being elements of the gauge algebra, and $\mathcal{D}$ is the generalized Dirac operator [6]. Putting $\mathcal{D}$ equal $\not \partial$ (or $D$ ), we can reproduce all properties of $\mathcal{A}$ (or $\Phi$ ), respectively. Therefore, the last terms of eqs. (4.1) and (4.2) can be combined with the gauge field that is being transformed, which guarantees that the result of the transformation on a gauge field is still a gauge field. On the other hand, depending on the symmetry group, one may or may not be able to write the $u\left[\Phi, u^{-1}\right]$ term in eq. (4.2) into the $a[D, b]$ form. In other words, due to the presence of the $u\left[\Phi, u^{-1}\right]$ term, the form of a gauge field defined as $\sum a[D, b]$ may not be closed after a gauge transformation. To maintain the gauge invariance, high order terms of the gauge field, arising from $u\left[\Phi, u^{-1}\right]$, are needed to build a covariant derivative. The requirement of vanishing $u\left[\Phi, u^{-1}\right]$ is the so called order-one condition [6].

It has been argued $[3,6]$ that the requirement of the order-one condition, together with a Majorana mass term for right handed neutrinos, selects the SM gauge group from $G_{422}=\mathrm{SU}(4) \times \mathrm{SU}(2)_{L} \times \mathrm{SU}(2)_{R}$. Furthermore, it has been shown [3] that if one starts with the $G_{422}=\mathrm{SU}(4) \times \mathrm{SU}(2)_{L} \times \mathrm{SU}(2)_{R}$ gauge group, and (partially) lifts the order-one condition, up to three models can be constructed. To be specific, starting with $G_{422}=$ $\mathrm{SU}(4) \times \mathrm{SU}(2)_{L} \times \mathrm{SU}(2)_{R}$, if the order-one condition is imposed on $G_{321}=\mathrm{SU}(3)_{C} \times$ $\mathrm{SU}(2)_{L} \times \mathrm{U}(1)_{Y}$, i.e. if the SM gauge transformation does not generate any higher order terms from $U\left[\Phi, U^{-1}\right]$ that cannot be written in the $\sum a[D, b]$ form, one ends with Model A of ref. [4]; if the order-one condition is lifted and if one only looks into a left-right asymmetric subset of the moduli space of the Dirac operator, one has Model B of ref. [4];

\footnotetext{
${ }^{9}$ Apparently, Leibniz rule is satisfied by the commutator. One might think that $[D, \cdot]$ cannot work as a derivation as it is not nilpotent in general. However, in the context of cycles over a $C^{*}$ algebra where integrals are defined using Hochschild cochains, $D$ does work as a derivation. For details on how to calculate such integrals, cf. refs. [5, 6].
} 
if the order-one condition is lifted and the full moduli space of the Dirac operator is considered, the result is Model $\mathrm{C}$ of ref. [4].

We note in passing that, if one starts with $G_{422}=\mathrm{SU}(4) \times \mathrm{SU}(2)_{L} \times \mathrm{SU}(2)_{R}$, it has been observed in [4] that the breaking of this gauge symmetry can be achieved either from usual Higgs mechanism as in refs. [1, 2], or from the use of the order-one condition [3, 72]. While it is possible that the the order-one condition encodes the symmetry breaking pattern, we refrain from discussing how the two seemingly different approaches could be related at some fundamental level to achieve similar results.

\subsection{The three models}

The three NCG models proposed in refs. [3, 4], which we refer to as models A, B, and $\mathrm{C}$, differ in their scalar sector content, and their unbroken symmetry structure is listed in table $1 .{ }^{10}$ As in our previous work $[1,2]$ we use the following notation for the symmetries:

$$
\begin{aligned}
G_{422 D} & =\mathrm{SU}(4)_{C} \otimes \mathrm{SU}(2)_{L} \otimes \mathrm{SU}(2)_{R} \otimes D, \\
G_{422} & =\mathrm{SU}(4)_{C} \otimes \mathrm{SU}(2)_{L} \otimes \mathrm{SU}(2)_{R}, \\
G_{3221} & =\mathrm{SU}(3)_{C} \otimes \mathrm{SU}(2)_{L} \otimes \mathrm{SU}(2)_{R} \otimes \mathrm{U}(1)_{B-L}, \\
G_{321} & =\mathrm{SU}(3)_{C} \otimes \mathrm{SU}(2)_{L} \otimes \mathrm{U}(1)_{Y}, \\
G_{31} & =\mathrm{SU}(3)_{C} \otimes \mathrm{U}(1)_{e m},
\end{aligned}
$$

where $D$ in $G_{422 D}$ refers to the left-right symmetry, a $Z_{2}$ symmetry which keeps the left and the right sectors equivalent. We adopt the normalization of the hypercharge $Y$ so that $Q_{e m}=I_{3}^{L}+Y=I_{3}^{L}+I_{3}^{R}+(B-L) / 2$. For comparison, the last row of table 1 lists the scalar content of an $\mathrm{SO}(10)$ based $G_{422}$ model studied in ref. [73], after $\mathrm{SO}(10)$ is broken to $G_{422}$.

When the $G_{422}$ (models A and B and $\mathrm{SO}(10)$ ) or $G_{442 D}$ (model C) of the NCG based models break to $G_{321}$ of the SM, the scalars listed in table 1 decompose into irreducible representations of $G_{321}$ as listed in the third column of table 2. Of the fields listed there, all the color-triplet fields have the quantum numbers of leptoquarks. For instance, the $\Delta_{R}(4,1,2)_{422}$ field which appears in model A decomposes as

$$
\begin{aligned}
& \Delta_{R}(4,1,2)_{422} \stackrel{G_{422} \rightarrow G_{3321}}{\longrightarrow} \widetilde{\Delta}_{R 1}(1,1,2,-1)_{3221}+\widetilde{\Delta}_{R 3}\left(3,1,2, \frac{1}{3}\right)_{3221} \\
& \stackrel{G_{3221} \rightarrow G_{321}}{\longrightarrow}\left[\widetilde{\Delta}_{R 1}(1,1,0)_{321}+\widetilde{\Delta}_{R 1}(1,1,-1)_{321}\right] \\
& +[\underbrace{\widetilde{\Delta}_{R 3}\left(3,1, \frac{2}{3}\right)_{321}}_{\bar{S}_{1}^{*}}+\underbrace{\widetilde{\Delta}_{R 3}\left(3,1,-\frac{1}{3}\right)_{321}}_{S_{1}^{*}}],
\end{aligned}
$$

\footnotetext{
${ }^{10}$ Recently, it has been argued that the incorporation of the Clifford structures in the spectral action formalism of the noncommutative geometry yields additional scalars as well [74]. The resulting model in that case turns out to be the Standard Model augmented by several scalars fields that carry the quantum numbers of the leptoquarks $\bar{S}_{1}$ and $\widetilde{R}_{2}$.
} 


\begin{tabular}{|l|l|l|}
\hline Model & Symmetry & Higgs Content \\
\hline \hline $\mathrm{A}$ & $G_{422}$ & $\phi(1,2,2)_{422}, \Sigma(15,1,1)_{422}, \widetilde{\Delta}_{R}(4,1,2)_{422}$ \\
\hline $\mathrm{B}$ & $G_{422}$ & $\phi(1,2,2)_{422}, \widetilde{\Sigma}(15,2,2)_{422}, \Delta_{R}(10,1,3)_{422}, H_{R}(6,1,1)_{422}$ \\
\hline $\mathrm{C}$ & $G_{422 D}$ & $\phi(1,2,2)_{422}, \widetilde{\Sigma}(15,2,2)_{422}, \Delta_{R}(10,1,3)_{422}, H_{R}(6,1,1)_{422}$, \\
& & $\Delta_{L}(10,3,1)_{422}, H_{L}(6,1,1)_{422}$ \\
\hline \hline $\mathrm{SO}(10)$ & $G_{422}$ & $\phi(1,2,2)_{422}, \Sigma(15,1,1)_{422}, \Delta_{R}(10,1,3)_{422}$ \\
\hline
\end{tabular}

Table 1. The scalar content of the three NCG based unified $G_{422}$ models proposed by Chamseddine, Connes, and van Suijlekom in refs. [3] and [4]. The last row lists for comparison the scalar content of the $\mathrm{SO}(10)$ based $G_{422}$ model discussed in ref. [73], below its unification scale where the $\mathrm{SO}(10)$ symmetry is broken to $G_{422}$. Note that in our notation throughout the paper the subscripts $L$ and $R$ of scalars indicate the chirality of fermions that the corresponding scalars couple to, which is shown explicitly in section 4 through the decomposition of the interaction terms, such as eqs. (4.26), (4.29), (4.35), and (4.37).

and we see that the two colored fields in the last line have quantum numbers corresponding to leptoquarks $\bar{S}_{1}$ and $S_{1}$. Fields with quantum numbers corresponding to the six leptoquarks $S_{1}, \widetilde{S}_{1}, \bar{S}_{1}, S_{3}, R_{2}$, and $\widetilde{R}_{2}$ all occur in one model or another. However, for any of these fields to be identifiable as leptoquarks, they must couple to quark-lepton pairs after symmetry breaking. In the following, we look at the scalar sectors of the three models one by one and identify the fields that can be considered leptoquarks. In particular, we will search for a field that can be identified as $S_{1}$ coupled to left-handed fermions.

\subsection{Couplings between fermions and scalars}

Following refs. $[3,4]$, we denote the $\mathrm{SU}(2)_{L}$ and $\mathrm{SU}(2)_{R}$ indices in the fundamental representation respectively with un-dotted and dotted lower-case Latin letters toward the beginning of the alphabet: e.g. $a=1,2$ and $\dot{a}=1,2$. Note that despite their appearance, these are NOT spinor indices, and so complex conjugation does not take on or off dots from the indices. ${ }^{11}$ The SU(4) index in the fundamental representation is denoted with upper-case Latin letters toward the middle of the alphabet: e.g. $I=0,1,2,3$, where $I=0$ is the lepton index and $I=i=1,2,3$ are the quark-color indices. ${ }^{12}$ The fermion content of the models is

$$
\begin{aligned}
& \psi_{a I}=\left(\psi_{a 0}, \psi_{a i}\right)=\left(\begin{array}{c}
\psi_{10}, \psi_{1 i} \\
\psi_{20}, \psi_{2 i}
\end{array}\right)=\left(L_{L}, Q_{L}\right)=\left(\begin{array}{c}
\nu_{L}, u_{L} \\
e_{L}, d_{L}
\end{array}\right), \\
& \psi_{\dot{a} I}=\left(\psi_{\dot{a} 0}, \psi_{\dot{a} i}\right)=\left(\begin{array}{c}
\psi_{\dot{1} 0}, \psi_{\dot{1} i} \\
\psi_{\dot{2} 0}, \psi_{\dot{2} i}
\end{array}\right)=\left(L_{R}, Q_{R}\right)=\left(\begin{array}{c}
\nu_{R}, u_{R} \\
e_{R}, d_{R}
\end{array}\right),
\end{aligned}
$$

\footnotetext{
${ }^{11}$ See, for instance, ref. [75].

${ }^{12}$ The authors of refs. [3,4] use $I=1,2,3,4$ to label the $\mathrm{SU}(4)$ index, and then use $I=(1, i)$, with $i=1,2,3$ to distinguish between the leptons and quarks. We instead use $I=0,1,2,3$, which seems more self-evident to us.
} 
that is, the SM content plus the right-handed neutrinos of each generation. The generation and spinor indices are suppressed. Complex (hermitian, Dirac) conjugation raises or lowers both indices, e.g.

$$
\bar{\psi}^{a I}=\overline{\psi_{a I}}, \quad \bar{\psi}^{\dot{a} I}=\overline{\psi_{\dot{a} I}} .
$$

In the case of the $\mathrm{SU}(2)$ 's, the index can be lowered or raised using

$$
(\epsilon)_{a b}, \quad\left(\epsilon^{\dagger}\right)^{a b}, \quad(\epsilon)_{\dot{a} \dot{b}}, \quad\left(\epsilon^{\dagger}\right)^{\dot{a} \dot{b}},
$$

where $\epsilon=i \sigma_{2}$.

The most general $G_{422}$ invariant Yukawa interaction in the NCG models involving $\psi_{a I}$ and $\psi_{\dot{a} I}$ can be written schematically as

$$
\mathcal{L}_{\mathrm{Y}}=\left(\bar{\psi}^{\dot{a} I} \gamma_{5} \Sigma_{\dot{a} I}^{b J} \psi_{b J}+\bar{\psi}_{a I}^{C} \gamma_{5} H^{a I b J} \psi_{b J}+\bar{\psi}_{\dot{a} I}^{C} \gamma_{5} H^{\dot{a} I \dot{b J}} \psi_{\dot{b} J}\right)+\text { h.c. },
$$

where $\psi^{C}=C \bar{\psi}^{T}$, and the couplings constants are embedded in the complex scalar fields $\Sigma_{\dot{a} I}^{b J}, H^{a I b J}$, and $H^{\dot{a} I \dot{b} J}$. The $\gamma_{5}$ that appears in this expression is due to the geometry being of even parity. The Hilbert space is $Z_{2}$ graded with $\gamma_{5}$ being the grading operator, so that the Dirac operator is of odd degree. This is crucial in order to interpret the discrete gauge field as the Higgs field. It is worth noting that, although seemingly originating from a different assumption, the superconnection formalism also captures this feature, as is shown in [76].

Since

$$
\overline{\psi_{1}^{C}} \gamma_{5} \psi_{2}=\overline{\psi_{2}^{C}} \gamma_{5} \psi_{1}
$$

for any pair of anti-commuting fermionic operators $\psi_{1}$ and $\psi_{2}$, the $H^{a I b J}$ and $H^{\dot{a} I \dot{b} J}$ fields are respectively symmetric under the interchange of the indices $(a I) \leftrightarrow(b J)$ and $(\dot{a} I) \leftrightarrow(\dot{b} J)$.

As is discussed above, the way scalars couple to fermions is dictated by the generalized gauge invariance. In eq. (4.8), $\Sigma_{\dot{a} I}^{b J}$ is the 'connection' that links chiral fermions to the ones with opposite chirality, i.e. it couples an $\mathrm{SU}(2)_{L}$ fermion to an $\mathrm{SU}(2)_{R}$ fermion. If a fermion is mapped to the same fermion with opposite chirality, a Dirac mass term can be generated after symmetry breaking. On the other hand, $H_{\dot{a} I \dot{I} . J}$ and $H_{a I b J}$ link fermions to anti-fermions with the same chirality. When a chiral fermion is mapped to its own charged conjugate, a Majorana mass term can be generated after symmetry breaking. Therefore, the former gives us $L R$ type coupling while the latter produces $R R$ and $L L$ types of coupling. This is how the Yukawa interaction terms are generated as a result of generalized gauge invariance. In particular, the NCG dictates there are only three terms, as indicated in eq. (4.8).

The complex scalar fields $\Sigma_{\dot{a} I}^{b J}, H_{\dot{a} I \dot{I} J}$, and $H_{a I b J}$ can, in general, consist of the following $G_{422}$ representations:

$$
\begin{aligned}
\Sigma_{\dot{a} I}^{b J} & =(1,2,2)_{422}+(15,2,2)_{422}, \\
H_{a I b J} & =(6,1,1)_{422}+(10,3,1)_{422}, \\
H_{\dot{a} I \dot{b} J} & =(6,1,1)_{422}+(10,1,3)_{422} .
\end{aligned}
$$


Note that 6 of $\mathrm{SU}(4)$ and 1 of $\mathrm{SU}(2)_{L}\left(\mathrm{SU}(2)_{R}\right)$ are respectively anti-symmetric under $I \leftrightarrow J$ and $a \leftrightarrow b(\dot{a} \leftrightarrow \dot{b})$ rendering the $(6,1,1)_{422}$ representation symmetric under $(a I) \leftrightarrow(b J)((\dot{a} I) \leftrightarrow(\dot{b} J))$. Note also that the leptoquark $S_{1}$ we seek must couple to lefthanded quarks and leptons, so we can expect to find such a field embedded in the complex scalar field $H_{a I b J}$.

Although the $G_{422}$ representations listed above are most general, and all the fields are contained in the model $\mathrm{C}$, the models $\mathrm{A}$ and $\mathrm{B}$ restrict the scalar sector to the fields listed in table 1. Since Model $\mathrm{C}$ is the only model which includes the field $H_{a I b J}$, it is clear that Model $\mathrm{C}$ is the place where we should be looking for our $S_{1}$ leptoquark. However, for the sake of completeness, we look at the couplings of the scalar fields that appear in all three models, and identify all the fields that correspond to one type of leptoquark or another.

\subsubsection{Model A}

In this model, the scalar field $H_{a I b J}$ is suppressed while the fields $\Sigma_{\dot{a} I}^{b J}$ and $H_{\dot{a} I \dot{b} J}$ are decomposed as

$$
\begin{aligned}
\Sigma_{\dot{a} I}^{b J} & =\left(k^{\nu} \phi_{\dot{a}}^{b}+k^{e} \widetilde{\phi}_{\dot{a}}^{b}\right) \Sigma_{I}^{J} / \Lambda+\left(k^{u} \phi_{\dot{a}}^{b}+k^{d} \widetilde{\phi}_{\dot{a}}^{b}\right)\left(\delta_{I}^{J}-\Sigma_{I}^{J} / \Lambda\right), \\
H_{\dot{a} I \dot{b} J} & =k^{* \nu_{R}} \Delta_{\dot{a} J} \Delta_{\dot{b} I} / \Lambda,
\end{aligned}
$$

where

$$
\widetilde{\phi}_{\dot{a}}^{b}=(\epsilon)_{\dot{a} \dot{c}} \bar{\phi}_{d}^{\dot{c}}\left(\epsilon^{\dagger}\right)^{d b}, \quad \epsilon=i \sigma_{2} .
$$

Note the manifest symmetry of $H_{\dot{a} I \dot{b} J}$ under $(\dot{a} I) \leftrightarrow(\dot{b} J)$. The fields $\phi_{\dot{a}}^{b}, \Sigma_{J}^{I}, \Delta_{\dot{a} J}$ are those labeled as $\phi(1,2,2)_{422}, \Sigma(15,1,1)_{422}$, and $\widetilde{\Delta}_{R}(4,1,2)_{422}$ in tables 1 and 2. Also, $k^{\nu}, k^{e}$, $k^{u}$, and $k^{d}$ denote Yukawa coupling matrices for the neutrinos, charged leptons, up-type quarks, and down-type quarks, respectively, while $k^{\nu_{R}}$ is the Majorana coupling matrix which gives Majorana masses to the right-handed neutrinos upon symmetry breaking, and the asterisk stands for complex conjugation. In order to give mass-dimension one to all scalar fields, we have introduced the scale $\Lambda$ which is only implicit in refs. [3, 4].

Note that the above decompositions render the interactions of the scalar fields $\widetilde{\Delta}_{R}(4,1,2)_{422}$ and $\Sigma(15,1,1)_{422}$ and the fermions into mass-dimension five operators, i.e. the generalized version of the $L H L H$ operator of the SM. To generate dimension-four operators of the Yukawa or Majorana type, we must give vacuum expectation values (VEV's) to the scalar fields. In the current case, VEV's are given to

$$
\left\langle\Sigma_{J}^{I}\right\rangle=\Lambda \delta_{0}^{I} \delta_{J}^{0}, \quad\left\langle\Delta_{\dot{a} J}\right\rangle=w \delta_{\dot{a}}^{\dot{1}} \delta_{J}^{0}, \quad\left\langle\phi_{\dot{a}}^{b}\right\rangle=v \delta_{\dot{a}}^{\dot{1}} \delta_{1}^{b}
$$

or in the notation of table 2 ,

$$
\left\langle\Sigma_{1}(1,1,0)_{321}\right\rangle=\Lambda, \quad\left\langle\widetilde{\Delta}_{R 1}(1,1,0)_{321}\right\rangle=w, \quad\left\langle\phi_{2}^{\prime}\left(1,2,-\frac{1}{2}\right)_{321}\right\rangle=\left[\begin{array}{l}
v \\
0
\end{array}\right] .
$$

Note that $\widetilde{\phi}_{\dot{a}}^{b}$ and $\phi_{\dot{a}}^{b}$ in eq. (4.11) are not independent so $\left\langle\phi_{\dot{a}}^{b}\right\rangle=v \delta_{\dot{a}}^{\dot{1}} \delta_{1}^{b}$ implies $\left\langle\widetilde{\phi}_{\dot{a}}^{b}\right\rangle=$ $v \delta_{\dot{a}}^{\dot{2}} \delta_{2}^{b}$. The VEV's $\Lambda$ and $w$ break $\mathrm{SU}(4) \times \mathrm{SU}(2)_{R}$ to $\mathrm{SU}(3) \times \mathrm{U}(1)_{Y}$, while $v$ breaks 
$\mathrm{SU}(2)_{L} \times \mathrm{U}(1)_{Y}$ down to $\mathrm{U}(1)_{e m}$. So symmetry breaking generates the following mass terms for the fermions:

$$
\begin{aligned}
& {\left[v\left(\bar{\psi}^{\mathrm{i} 0} k^{\nu} \psi_{10}+\bar{\psi}^{20} k^{e} \psi_{20}\right)+v\left(\bar{\psi}^{\mathrm{i} i} k^{u} \psi_{1 i}+\bar{\psi}^{2 i} k^{d} \psi_{2 i}\right)+\frac{w^{2}}{\Lambda} \overline{\psi^{C}}{ }_{i 0} k^{\nu_{R}} \psi_{10}\right]+\text { h.c. }} \\
& =\left[v\left(\overline{\nu_{R}} k^{\nu} \nu_{L}+\overline{e_{R}} k^{e} e_{L}\right)+v\left(\overline{u_{R}} k^{u} u_{L}+\overline{d_{R}} k^{d} d_{L}\right)+\frac{w^{2}}{\Lambda} \overline{\nu_{R}^{C}} k^{\nu_{R}} \nu_{R}\right]+\text { h.c. }
\end{aligned}
$$

The couplings of the $\phi(1,2,2)_{422}, \widetilde{\Delta}_{R}(4,1,2)_{422}$, and $\Sigma(15,1,1)_{422}$ fields to the fermions can be obtained by substituting eq. (4.11) into the generic expression eq. (4.8), taking into account eq. (4.13). First, let us take $\Delta_{\dot{a} I}=\widetilde{\Delta}_{R}(4,1,2)_{422}$ as an example:

$$
\begin{aligned}
& \bar{\psi}_{\dot{a} I} \gamma_{5} H^{\dot{a} I \dot{b} J} \psi_{\dot{b} J}+\text { h.c. } \\
& =\frac{1}{\Lambda} \overline{\psi^{C}}{ }_{\dot{a} I} \Delta^{\dot{b} I} k^{\nu_{R}} \Delta^{\dot{a} J} \psi_{\dot{b} J}+\text { h.c. } \\
& =\frac{1}{\Lambda}\left[\left(\overline{L_{R}^{C}} \widetilde{\Delta}_{R 1}^{*}\right) k^{\nu_{R}}\left(\widetilde{\Delta}_{R 1}^{*} L_{R}\right)+\left(\overline{Q_{R}^{C}} \widetilde{\Delta}_{R 1}^{*}\right) k^{\nu_{R}}\left(\widetilde{\Delta}_{R 3}^{*} L_{R}\right)\right. \\
& \left.\quad+\left(\overline{L_{R}^{C}} \widetilde{\Delta}_{R 3}^{*}\right) k^{\nu_{R}}\left(\widetilde{\Delta}_{R 1}^{*} Q_{R}\right)+\left(\overline{Q_{R}^{C}} \widetilde{\Delta}_{R 3}^{*}\right) k^{\nu_{R}}\left(\widetilde{\Delta}_{R 3}^{*} Q_{R}\right)\right]+ \text { h.c. },
\end{aligned}
$$

where the lines connecting the colored fields indicate color contraction, and we have also used the shorthand

$$
\begin{aligned}
& \widetilde{\Delta}_{R 1}=\widetilde{\Delta}_{R 1}(1,1,2,-1)_{3221}=\left[\begin{array}{l}
\widetilde{\Delta}_{R 1}(1,1,0)_{321} \\
\widetilde{\Delta}_{R 1}(1,1,-1)_{321}
\end{array}\right], \\
& \widetilde{\Delta}_{R 3}=\widetilde{\Delta}_{R 3}\left(3,1,2, \frac{1}{3}\right)_{3221}=\left[\begin{array}{l}
\widetilde{\Delta}_{R 3}\left(3,1,+\frac{2}{3}\right)_{321} \\
\widetilde{\Delta}_{R 3}\left(3,1,-\frac{1}{3}\right)_{321}
\end{array}\right],
\end{aligned}
$$

for the fields that $\widetilde{\Delta}(4,1,2)_{422}$ decomposes into as $G_{422} \rightarrow G_{3221} \rightarrow G_{321}$. After the SM singlet $\widetilde{\Delta}_{R 1}(1,1,0)_{321}$ develops a VEV, eq. (4.14), we find

$$
\begin{aligned}
& \overline{\psi^{C}}{ }_{\dot{a} I} \gamma_{5} H^{\dot{a} I \dot{b} J} \psi_{\dot{b} J}+\text { h.c. } \\
& \rightarrow \frac{2 w}{\Lambda}\left[\frac{w}{2}\left(\overline{\nu_{R}^{C}} k^{\nu_{R}} \nu_{R}\right)+\left(\overline{\nu_{R}^{C}} k^{\nu_{R}} \nu_{R}\right) \widetilde{\Delta}_{R 1}^{*(0)}+\left(\overline{\nu_{R}^{C}} k^{\nu_{R}} e_{R}\right) \widetilde{\Delta}_{R 1}^{*(1)}\right. \\
& +\left(\overline{u_{R}^{C}} k^{\nu_{R}} \nu_{R}\right) \underbrace{\widetilde{\Delta}_{R 3}^{*(-2 / 3)}}_{\bar{S}_{1}^{(-2 / 3)}}+\left(\overline{u_{R}^{C}} k^{\nu_{R}} e_{R}\right) \underbrace{\widetilde{\Delta}_{R 3}^{*(1 / 3)}}_{S_{1}^{(1 / 3)}}+\cdots]+ \text { h.c. }
\end{aligned}
$$

where the first term inside the brackets is the Majorana mass term for the right-handed neutrinos, the ellipses represent dimension 5 operators, and we have used the symbols

$$
\begin{aligned}
& \widetilde{\Delta}_{R 1}^{*(0)}=\left[\widetilde{\Delta}_{R 1}(1,1,0)_{321}\right]^{*}-w \\
& \widetilde{\Delta}_{R 1}^{*(1)}=\left[\widetilde{\Delta}_{R 1}(1,1,-1)_{321}\right]^{*}, \\
& \widetilde{\Delta}_{R 3}^{*(-2 / 3)}=\left[\widetilde{\Delta}_{R 3}\left(3,1,+\frac{2}{3}\right)_{321}\right]^{*}, \\
& \widetilde{\Delta}_{R 3}^{*(1 / 3)}=\left[\widetilde{\Delta}_{R 3}\left(3,1,-\frac{1}{3}\right)_{321}\right]^{*} .
\end{aligned}
$$


Thus, we find that $\widetilde{\Delta}_{R}(4,1,2)_{422}$ includes the leptoquarks $S_{1}$ and $\bar{S}_{1}$, but this $S_{1}$, by construction, only couples to $u_{R}$ and $e_{R}$. Note also that it does not couple to diquarks.

Next, let us look at the couplings of $\phi_{\dot{a}}^{b}=\phi(1,2,2)_{422}$ and $\Sigma_{I}^{J}=\Sigma(15,1,1)_{422}$ :

$$
\begin{aligned}
& \bar{\psi}^{\dot{a} I} \gamma_{5} \Sigma_{\dot{a} I}^{b J} \psi_{b J}+\text { h.c. } \\
& =\bar{\psi}^{\dot{a} I} \gamma_{5}\left[\left(k^{\nu} \phi_{\dot{a}}^{b}+k^{e} \widetilde{\phi}_{\dot{a}}^{b}\right) \Sigma_{I}^{J} / \Lambda+\left(k^{u} \phi_{\dot{a}}^{b}+k^{d} \widetilde{\phi}_{\dot{a}}^{b}\right)\left(\delta_{I}^{J}-\Sigma_{I}^{J} / \Lambda\right)\right] \psi_{b J}+\text { h.c. }
\end{aligned}
$$

Note that if $k^{u}=k^{\nu}$ and $k^{d}=k^{e}$, then $\Sigma_{I}^{J}$ will decouple from the fermions. We are interested in the couplings of $\Sigma_{I}^{J}=\Sigma(15,1,1)_{422}$ to the fermions so we replace $\phi_{\dot{a}}^{b}$ and $\widetilde{\phi}_{\dot{a}}^{b}$ with their VEV's, and denote the shifted $\Sigma(15,1,1)_{422}$ fields as

$$
\begin{aligned}
\Sigma_{0}^{0} & =\Sigma_{1}(1,1,0)_{321}=\Sigma_{1}^{(0)}=\Lambda+\Sigma_{1}^{(0)}, \\
\Sigma_{i}^{0} & =\Sigma_{3}\left(3,1,+\frac{2}{3}\right)_{321}=\Sigma_{3}^{(2 / 3)} \\
\Sigma_{0}^{j} & =\Sigma_{\overline{3}}\left(\overline{3}, 1,-\frac{2}{3}\right)_{321}=\Sigma_{\overline{3}}^{(-2 / 3)} \\
\Sigma_{i}^{j} & =\Sigma_{8}(8,1,0)_{321}=\Sigma_{8}^{(0)} .
\end{aligned}
$$

We find:

$$
\begin{aligned}
\bar{\psi}^{\dot{a} I} \gamma_{5}\left[\left(k^{\nu} \phi_{\dot{a}}^{b}+k^{e} \widetilde{\phi}_{\dot{a}}^{b}\right) \Sigma_{I}^{J} / \Lambda+\left(k^{u} \phi_{\dot{a}}^{b}+k^{d} \widetilde{\phi}_{\dot{a}}^{b}\right)\left(\delta_{I}^{J}-\Sigma_{I}^{J} / \Lambda\right)\right] \psi_{b J}+\text { h.c. } \\
\rightarrow-v\left[\left(\bar{\psi}^{i I} k^{\nu} \psi_{1 J}+\bar{\psi}^{2 I} k^{e} \psi_{2 J}\right) \Sigma_{I}^{J} / \Lambda+\left(\bar{\psi}^{i} k^{u} \psi_{1 J}+\bar{\psi}^{2 I} k^{d} \psi_{2 J}\right)\left(\delta_{I}^{J}-\Sigma_{I}^{J} / \Lambda\right)\right]+\text { h.c. } \\
=-v\left[\left(\overline{\nu_{R}} k^{\nu} \nu_{L}+\overline{e_{R}} k^{e} e_{L}\right)+\left(\overline{u_{R}} k^{u} u_{L}+\overline{d_{R}} k^{d} d_{L}\right)\right] \\
+\frac{v}{\Lambda}\left[\left\{\overline{\nu_{R}}\left(\delta k^{+}\right) \nu_{L}+\overline{e_{R}}\left(\delta k^{-}\right) e_{L}\right\} \Sigma_{1}^{\prime(0)}+\left\{\overline{u_{R}}\left(\delta k^{+}\right) u_{L}+\overline{d_{R}}\left(\delta k^{-}\right) d_{L}\right\} \Sigma_{8}^{(0)}\right. \\
\left.\quad+\left\{\overline{\nu_{R}}\left(\delta k^{+}\right) u_{L}+\overline{e_{R}}\left(\delta k^{-}\right) d_{L}\right\} \Sigma_{\overline{3}}^{(-2 / 3)}+\left\{\overline{u_{R}}\left(\delta k^{+}\right) \nu_{L}+\overline{d_{R}}\left(\delta k^{-}\right) e_{L}\right\} \Sigma_{3}^{(2 / 3)}\right] \\
\quad+\text { h.c. }
\end{aligned}
$$

where

$$
\delta k^{+} \equiv k^{u}-k^{\nu}, \quad \delta k^{-} \equiv k^{d}-k^{e} .
$$

Comparing with eq. (A.11), we can see that though $\Sigma_{I}^{J}=\Sigma(15,1,1)_{422}$ does not include fields corresponding to the leptoquark doublets $R_{2}$ and $\widetilde{R}_{2}$, its components $\Sigma_{3}^{(2 / 3)}$ and $\Sigma_{\overline{3}}^{(-2 / 3)}$ do correspond to the following leptoquark components after the symmetry breaking $G_{321} \rightarrow G_{31}:$

$$
\begin{array}{lll}
\Sigma_{3}^{(2 / 3)} & \leftrightarrow R_{2}^{(2 / 3)}, & \widetilde{R}_{2}^{(2 / 3)}, \\
\Sigma_{\overline{3}}^{(-2 / 3)} & \leftrightarrow R_{2}^{*(2 / 3)}, \widetilde{R}_{2}^{*(2 / 3)},
\end{array}
$$

with their couplings determined by the Yukawa coupling matrices $\delta k^{+}=k^{u}-k^{\nu}$ and $\delta k^{-}=k^{d}-k^{e}$. These fields also do not couple to diquarks. 


\subsubsection{Model B}

In Model B, the field $H_{a I b J}$ is still suppressed, whereas the fields $\Sigma_{\dot{a} I}^{b J}$ and $H_{\dot{a} I \dot{b} J}$ are no longer decomposed as shown in eq. (4.11). They are treated as mass-dimension one operators, i.e. 'fundamental' in the language of ref. [3].

Although $H_{\dot{a} I \dot{b} J}$ is treated as a dimension-one field, it is still a product representation $(4,1,2) \times(4,1,2)=(10,1,3)+(6,1,1)$. Recall the symmetry of $H_{\dot{a} I \dot{b} J}$ under $(\dot{a} I) \leftrightarrow(\dot{b} J)$. We can write

$$
H_{\dot{a} I \dot{b} J}=\underbrace{\frac{1}{4}\left(H_{\dot{a} I \dot{b} J}+H_{\dot{b} I \dot{a} J}+H_{\dot{b} J \dot{a} I}+H_{\dot{a} J \dot{b} I}\right)}_{=\Delta_{(\dot{a} \dot{b})(I J)}=\Delta_{R}(10,1,3)_{422}}+\underbrace{\frac{1}{4}\left(H_{\dot{a} I \dot{b} J}-H_{\dot{b} I \dot{a} J}+H_{\dot{b} J \dot{a} I}-H_{\dot{a} J \dot{b} I}\right)}_{=H_{[\dot{a} \dot{b}][I J]}=H_{R}(6,1,1)_{422}},
$$

where the parentheses and brackets on the indices respectively indicate symmetrization and anti-symmetrization. Let us look at the couplings of the two terms separately. First, $\Delta_{(\dot{a} \dot{b})(I J)}=\Delta_{R}(10,1,3)_{422}$ :

$$
\begin{aligned}
& \bar{\psi}_{\dot{a} I} \gamma_{5} \Delta^{(\dot{a} \dot{b})(I J)} \psi_{\dot{b} J}+\text { h.c. } \\
& =\left(\bar{\psi}_{\dot{a} 0}^{C} \Delta^{(\dot{a} \dot{b})(00)} \psi_{\dot{b} 0}+\bar{\psi}_{\dot{a} 0}^{C} \Delta^{(\dot{a} \dot{b})(0 j)} \psi_{\dot{b} j}+{\overline{\psi^{C}}}_{\dot{a} i} \Delta^{(\dot{a} \dot{b})(i 0)} \psi_{\dot{b} 0}+\bar{\psi}_{\dot{a} i}^{C} \Delta^{(\dot{a} \dot{b})(i j)} \psi_{\dot{b} j}\right)+\text { h.c. } \\
& =\left[\left(\bar{L}_{R}^{C} \epsilon \vec{\tau} L_{R}\right) \vec{\Delta}_{R 1}^{*}+2\left(\overline{Q_{R}^{C}} \epsilon \vec{\tau} L_{R}\right) \vec{\Delta}_{R 3}^{*}+\left(\overline{Q_{R}^{C}} \epsilon \vec{\tau} Q_{R}\right) \vec{\Delta}_{R 6}^{*}\right]+\text { h.c. }
\end{aligned}
$$

where we use the shorthand

$$
\begin{aligned}
& \vec{\Delta}_{R 1}=\Delta_{(\dot{a} \dot{b})(00)}=\Delta_{R 1}(1,1,3,-2)_{3221}=\left[\begin{array}{l}
\Delta_{R 1}(1,1,0)_{321} \\
\Delta_{R 1}(1,1,-1)_{321} \\
\Delta_{R 1}(1,1,-2)_{321}
\end{array}\right], \\
& \vec{\Delta}_{R 3}=\Delta_{(\dot{a} \dot{b})(0 j)}=\Delta_{R 3}\left(3,1,3,-\frac{2}{3}\right)_{3221}=\left[\begin{array}{c}
\Delta_{R 3}\left(3,1, \frac{2}{3}\right)_{321} \\
\Delta_{R 3}\left(3,1,-\frac{1}{3}\right)_{321} \\
\Delta_{R 3}\left(3,1,-\frac{4}{3}\right)_{321}
\end{array}\right],
\end{aligned}
$$

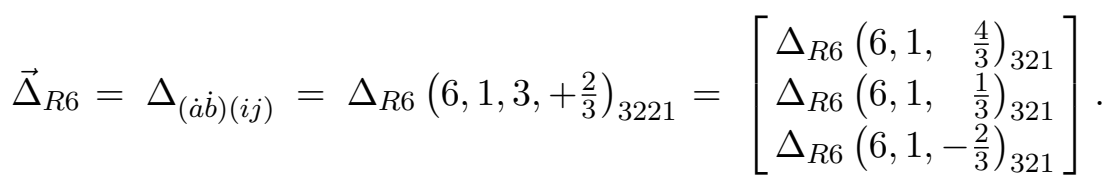

The leptoquark fields are $\vec{\Delta}_{R 3}$. Expanding the interaction, we find

$$
\begin{aligned}
& 2\left(\overline{Q_{R}^{C}} \epsilon \vec{\tau} L_{R}\right) \vec{\Delta}_{R 3}^{*}+\text { h.c. } \\
& =[\sqrt{2}\left(\overline{u_{R}^{C}} \nu_{R}\right) \underbrace{\Delta_{R 3}^{*(-2 / 3)}}_{\bar{S}_{1}^{(-2 / 3)}}+\left(\overline{u_{R}^{C}} e_{R}+\overline{d_{R}^{C}} \nu_{R}\right) \underbrace{\Delta_{R 3}^{*(1 / 3)}}_{S_{1}^{(1 / 3)}}+\sqrt{2}\left(\overline{d_{R}^{C}} e_{R}\right) \underbrace{\Delta_{R 3}^{*(4 / 3)}}_{\widetilde{S}_{1}^{(4 / 3)}}]+\text { h.c. }
\end{aligned}
$$

Thus, the field $\Delta_{R}(10,1,3)_{422}$ includes components corresponding to the leptoquarks $S_{1}$, $\widetilde{S}_{1}$, and $\bar{S}_{1}$. They all couple only to right-handed fermions by construction, and none of them couple to diquarks. 
Next, let us consider the couplings of $H_{[\dot{a} \dot{b}][I J]}=H_{R}(6,1,1)_{422}$. We find:

$$
\begin{aligned}
& \bar{\psi}_{\dot{a} I} \gamma_{5} H^{[\dot{a} \dot{b}][I J]} \psi_{\dot{b} J}+\text { h.c. } \\
& =\left[\left(\overline{\psi^{C}} \psi_{\dot{a} 0}-\overline{\psi^{C}}{ }_{\dot{a} j} \psi_{\dot{b} 0}\right) H^{[\dot{a} \dot{b}][0 j]}+\overline{\psi^{C}} \psi_{\dot{a} i} \psi_{\dot{b} j} H^{[\dot{a} \dot{b}][i j]}\right]+\text { h.c. } \\
& =\left[\left(\overline{L_{R}^{C}} \epsilon Q_{R j}-\overline{Q_{R j}^{C}} \epsilon L_{R}\right) H^{[\dot{1} \dot{2}][0 j]}+\left(\overline{Q_{R i}^{C}} \epsilon Q_{R j}\right) H^{[\dot{i} \dot{2}][i j]}\right]+\text { h.c. } \\
& =2[\left(\overline{d_{R j}^{C}} \nu_{R}-\overline{u_{R j}^{C}} e_{R}\right) \underbrace{H_{3 R}^{* j}}_{S_{1}^{(1 / 3)}}+\varepsilon^{i j k} \overline{u_{R i}^{C}} d_{R j} H_{\overline{3} R k}^{*}]+\text { h.c. }
\end{aligned}
$$

where in the last line we have set

$$
\begin{aligned}
H_{3 R j} & =H_{[i \dot{2}][0, j]}, \\
H_{\overline{3} R}{ }^{k} & =\frac{1}{2} \varepsilon^{i j k} H_{[i \dot{2}][i, j]} \quad \rightarrow \quad H_{[i \dot{2}][i, j]}=\varepsilon_{i j k} H_{\overline{3} R}{ }^{k} .
\end{aligned}
$$

So $H_{3 R}\left(3,1,-\frac{1}{3}\right)_{321}$ corresponds to leptoquark $S_{1}^{*}$ coupled to right-handed fermions. The field $H_{\overline{3} R}\left(\overline{3}, 1, \frac{1}{3}\right)_{321}$ has the quantum numbers of $S_{1}$, but it only couples to diquarks.

Now let us look at the other field $\Sigma_{\dot{a} I}^{b J}$. It is also a product representation, $(\overline{4}, 2,1) \times(4,1,2)=(1,2,2)+(15,2,2)$. The corresponding fields are denoted $\phi(1,2,2)_{422}$ and $\widetilde{\Sigma}(15,2,2)_{422}$. The couplings of $\phi_{\dot{a}}^{b}=\phi(1,2,2)_{422}$ to the fermions are simply

$$
\bar{\psi}^{\dot{a} I} \gamma_{5}\left(k^{u} \phi_{\dot{a}}^{b}+k^{d} \tilde{\phi}_{\dot{a}}^{b}\right) \psi_{b I}+\text { h.c. }
$$

where $k^{u, d}$ are Yukawa coupling matrices, which will give the same masses to the quarks and leptons upon symmetry breaking to $G_{31}$. This quark-lepton symmetry is broken by the couplings to $\widetilde{\Sigma}_{\dot{a} I}^{b J}=\widetilde{\Sigma}(15,2,2)_{422}$. They read:

$$
\begin{aligned}
& \left(\bar{\psi}^{\dot{a} I} \gamma_{5} \widetilde{\Sigma}_{\dot{a} I}^{b J} \psi_{b J}\right)+\text { h.c. } \\
& =-\left(\bar{\psi}^{\dot{a} 0} \widetilde{\Sigma}_{\dot{a} 0}^{b 0} \psi_{b 0}+\bar{\psi}^{\dot{a} i} \widetilde{\Sigma}_{\dot{a} i}^{b 0} \psi_{b 0}+\bar{\psi}^{\dot{a} 0} \widetilde{\Sigma}_{\dot{a} 0}^{b j} \psi_{b j}+\bar{\psi}^{\dot{a} i} \widetilde{\Sigma}_{\dot{a} i}^{b j} \psi_{b j}\right)+\text { h.c. } \\
& =-\left(\overline{L_{R}} \widetilde{\Sigma}_{1}^{\dagger} L_{L}+\overline{Q_{R}} \widetilde{\Sigma}_{\overline{3}}^{\dagger} L_{L}+\overline{L_{R}} \widetilde{\Sigma}_{3}^{\dagger} Q_{L}+\overline{Q_{R}} \widetilde{\Sigma}_{8}^{\dagger} Q_{L}\right)+\text { h.c. }
\end{aligned}
$$

where

$$
\begin{aligned}
& \widetilde{\Sigma}_{1}=\widetilde{\Sigma}_{1}(1,2,2,0)_{3221}=\left[\begin{array}{ll}
\widetilde{\Sigma}_{1}^{\prime}\left(1,2,-\frac{1}{2}\right)_{321} & \widetilde{\Sigma}_{1}\left(1,2, \frac{1}{2}\right)_{321}
\end{array}\right]=\left[\begin{array}{ll}
\widetilde{\Sigma}_{1}^{\prime(0)} & \widetilde{\Sigma}_{1}^{(1)} \\
\widetilde{\Sigma}_{1}^{\prime(-1)} & \widetilde{\Sigma}_{1}^{(0)}
\end{array}\right], \\
& \widetilde{\Sigma}_{3}=\widetilde{\Sigma}_{3}\left(3,2,2,+\frac{4}{3}\right)_{3221}=\left[\begin{array}{ll}
\widetilde{\Sigma}_{3}^{\prime}\left(3,2,+\frac{1}{6}\right)_{321} & \widetilde{\Sigma}_{3}\left(3,2,+\frac{7}{6}\right)_{321}
\end{array}\right]=\left[\begin{array}{ll}
\widetilde{\Sigma}_{3}^{\prime(2 / 3)} & \widetilde{\Sigma}_{3}^{(5 / 3)} \\
\widetilde{\Sigma}_{3}^{\prime(-1 / 3)} & \widetilde{\Sigma}_{3}^{(2 / 3)}
\end{array}\right], \\
& \widetilde{\Sigma}_{\overline{3}}=\widetilde{\Sigma}_{\overline{3}}\left(\overline{3}, 2,2,-\frac{4}{3}\right)_{3221}=\left[\begin{array}{lll}
\widetilde{\Sigma}_{\overline{3}}^{\prime}\left(\overline{3}, 2,-\frac{7}{6}\right)_{321} & \widetilde{\Sigma}_{\overline{3}}\left(\overline{3}, 2,-\frac{1}{6}\right)_{321}
\end{array}\right]=\left[\begin{array}{ll}
\widetilde{\Sigma}_{\overline{3}}^{\prime(-2 / 3)} & \widetilde{\Sigma}_{\overline{3}}^{(1 / 3)} \\
\widetilde{\Sigma}_{\overline{3}}^{\prime(-5 / 3)} & \widetilde{\Sigma}_{\overline{3}}^{(-2 / 3)}
\end{array}\right], \\
& \widetilde{\Sigma}_{8}=\widetilde{\Sigma}_{8}(8,2,2,0)_{3221}=\left[\begin{array}{lll}
\widetilde{\Sigma}_{8}^{\prime}\left(8,2,-\frac{1}{2}\right)_{321} & \widetilde{\Sigma}_{8}(8,2, & \left.\frac{1}{2}\right)_{321}
\end{array}\right]=\left[\begin{array}{ll}
\widetilde{\Sigma}_{8}^{\prime(0)} & \widetilde{\Sigma}_{8}^{(1)} \\
\widetilde{\Sigma}_{8}^{\prime(-1)} & \widetilde{\Sigma}_{8}^{(0)}
\end{array}\right] .
\end{aligned}
$$


Only the uncolored field $\widetilde{\Sigma}_{1}$ can develop a VEV upon breaking to $G_{31}$, which will only give masses to the leptons and break quark-lepton universality. The leptoquark fields are $\widetilde{\Sigma}_{3}$ and $\widetilde{\Sigma}_{\overline{3}}$ : Expanding out the couplings explicitly, we find:

$$
\begin{aligned}
& \overline{Q_{R}} \widetilde{\Sigma}_{\overline{3}}^{\dagger} L_{L}=[\left(\overline{u_{R}} \nu_{L}\right) \underbrace{\widetilde{\Sigma}_{\overline{3}}^{*(2 / 3)}}_{R_{2}^{(2 / 3)}}+\left(\overline{u_{R}} e_{L}\right) \underbrace{\widetilde{\Sigma}_{\overline{3}}^{*(5 / 3)}}_{R_{2}^{(5 / 3)}}+\left(\overline{d_{R}} \nu_{L}\right) \underbrace{\widetilde{\Sigma}_{\overline{3}}^{*(-1 / 3)}}_{\widetilde{R}_{2}^{(-1 / 3)}}+\left(\overline{d_{R}} e_{L}\right) \underbrace{\widetilde{\Sigma}_{\overline{3}}^{*(2 / 3)}}_{\widetilde{R}_{2}^{(2 / 3)}}], \\
& \overline{L_{R}} \widetilde{\Sigma}_{3}^{\dagger} Q_{L}=[\left(\overline{\nu_{R}} u_{L}\right) \underbrace{\widetilde{\Sigma}_{3}^{*(-2 / 3)}}_{\widetilde{R}_{2}^{*(-2 / 3)}}+\left(\overline{\nu_{R}} d_{L}\right) \underbrace{\widetilde{\Sigma}_{3}^{*(1 / 3)}}_{\widetilde{R}_{2}^{*(1 / 3)}}+\left(\overline{e_{R}} u_{L}\right) \underbrace{\widetilde{\Sigma}_{3}^{*(-5 / 3)}}_{R_{2}^{*(-5 / 3)}}+\left(\overline{e_{R}} d_{L}\right) \underbrace{\widetilde{\Sigma}_{3}^{*(-2 / 3)}}_{R_{2}^{*(-2 / 3)}}] .
\end{aligned}
$$

Note that though both $\widetilde{\Sigma}_{\overline{3}}^{*(2 / 3)}$ and $\widetilde{\Sigma}_{3}^{(2 / 3)}$, for instance, have the quantum numbers and couplings of the leptoquark $R_{2}^{(2 / 3)}$, they are independent fields. Note also that these leptoquarks do not couple to diquarks.

\subsubsection{Model C}

As was discussed above, Model C is the left-right symmetrization of Model B. Besides all the fields listed in Model B that couple to right-handed fermions, it has the counterpart scalars that couple to left-handed fermions, $H_{a I b J}=\Delta_{(a b)(I J)}+H_{[a b][I J]}=\Delta_{L}(10,3,1)_{422}+$ $H_{L}(6,1,1)_{422}$, in addition to $H_{\dot{a} I \dot{b} J}=\Delta_{(\dot{a} \dot{b})(I J)}+H_{[\dot{a} \dot{b}][I J]}=\Delta_{R}(10,1,3)_{422}+H_{R}(6,1,1)_{422}$. The couplings of $\Delta_{L}(10,3,1)_{422}$ and $H_{L}(6,1,1)_{422}$ to the fermions can be obtained from those of $\Delta_{R}(10,1,3)_{422}$ and $H_{R}(6,1,1)_{422}$, eqs. (4.26), (4.28), and (4.29), by simply replacing the right-handed fermions with their left-handed counterparts. First, the couplings of $\Delta_{L}(10,3,1)_{422}$ are

$$
\begin{aligned}
& \bar{\psi}_{a I} \gamma_{5} \Delta^{(a b)(I J)} \psi_{b J}+\text { h.c. } \\
& =[\left(\overline{L_{L}^{C}} \epsilon \vec{\tau} L_{L}\right) \vec{\Delta}_{L 1}^{*}+2\left(\overline{Q_{L}^{C}} \epsilon \vec{\tau} L_{L}\right) \underbrace{\vec{\Delta}_{L 3}^{*}}_{\vec{S}_{3}}+\left(\overline{Q_{L}^{C}} \epsilon \vec{\tau} Q_{L}\right) \vec{\Delta}_{L 6}^{*}]+\text { h.c. }
\end{aligned}
$$

Expanding the $\vec{\Delta}_{L 3}^{*}$ term we obtain

$$
\begin{aligned}
& 2\left(\overline{Q_{L}^{C}} \epsilon \vec{\tau} L_{L}\right) \vec{\Delta}_{L 3}^{*}+\text { h.c. } \\
& =[\sqrt{2}\left(\overline{u_{L}^{C}} \nu_{L}\right) \underbrace{\Delta_{L 3}^{*(-2 / 3)}}_{S_{3}^{(-2 / 3)}}+\left(\overline{u_{L}^{C}} e_{L}+\overline{d_{L}^{C}} \nu_{L}\right) \underbrace{\Delta_{L 3}^{*(1 / 3)}}_{S_{3}^{(1 / 3)}}+\sqrt{2}\left(\overline{d_{L}^{C}} e_{L}\right) \underbrace{\Delta_{L 3}^{*(4 / 3)}}_{S_{3}^{(4 / 3)}}]+\text { h.c. }
\end{aligned}
$$

Thus, we obtain the isotriplet leptoquark $S_{3}$. The couplings of $H_{L}(6,1,1)_{422}$ on the other hand are

$$
\begin{aligned}
& \overline{\psi^{C}}{ }_{a I} \gamma_{5} H^{[a b][I J]} \psi_{b J}+\text { h.c. } \\
& =2[\left(\overline{d_{L j}^{C}} \nu_{L}-\overline{u_{L j}^{C}} e_{L}\right) \underbrace{H_{3 L}^{* j}}_{S_{1}^{(1 / 3)}}+\varepsilon^{i j k} \overline{u_{L i}^{C}} d_{L j} H_{\overline{3} L k}^{*}]+\text { h.c. }
\end{aligned}
$$




\begin{tabular}{|c|c|c|}
\hline$G_{422}$ & $G_{3221}$ & $G_{321}$ \\
\hline$\phi(1,2,2)_{422}$ & $\phi(1,2,2,0)_{3221}$ & $\phi_{2}\left(1,2, \frac{1}{2}\right)_{321}, \phi_{2}^{\prime}\left(1,2,-\frac{1}{2}\right)_{321}$ \\
\hline \multirow{2}{*}{$\widetilde{\Delta}_{R}(4,1,2)_{422}$} & $\widetilde{\Delta}_{R 1}(1,1,2,-1)_{3221}$ & $\widetilde{\Delta}_{R 1}(1,1,0)_{321}, \widetilde{\Delta}_{R 1}(1,1,-1)_{321}$ \\
\hline & $\widetilde{\Delta}_{R 3}\left(3,1,2, \frac{1}{3}\right)_{3221}$ & $\widetilde{\Delta}_{R 3}\left(3,1, \frac{2}{3}\right)_{321}, \widetilde{\Delta}_{R 3}\left(3,1,-\frac{1}{3}\right)_{321}$ \\
\hline \multirow[t]{3}{*}{$\Delta_{R}(10,1,3)_{422}$} & $\Delta_{R 1}(1,1,3,-2)_{3221}$ & $\Delta_{R 1}(1,1,0)_{321}, \Delta_{R 1}(1,1,-1)_{321}, \Delta_{R 1}(1,1,-2)_{321}$ \\
\hline & $\Delta_{R 3}\left(3,1,3,-\frac{2}{3}\right)_{3221}$ & $\Delta_{R 3}\left(3,1, \frac{2}{3}\right)_{321}, \Delta_{R 3}\left(3,1,-\frac{1}{3}\right)_{321}, \Delta_{R 3}\left(3,1,-\frac{4}{3}\right)_{321}$ \\
\hline & $\Delta_{R 6}\left(6,1,3, \frac{2}{3}\right)_{3221}$ & $\Delta_{R 6}\left(6,1, \frac{4}{3}\right)_{321}, \Delta_{R 6}\left(6,1, \frac{1}{3}\right)_{321}, \Delta_{R 6}\left(6,1,-\frac{2}{3}\right)_{321}$ \\
\hline \multirow{3}{*}{$\Delta_{L}(10,3,1)_{422}$} & $\Delta_{L 1}(1,3,1,-2)_{3221}$ & $\Delta_{L 1}(1,3,-1)_{321}$ \\
\hline & $\Delta_{L 3}\left(3,3,1,-\frac{2}{3}\right)_{3221}$ & $\Delta_{L 3}\left(3,3,-\frac{1}{3}\right)_{321}$ \\
\hline & $\Delta_{L 6}\left(6,3,1, \frac{2}{3}\right)_{3221}$ & $\Delta_{L 6}\left(6,3, \frac{1}{3}\right)_{321}$ \\
\hline \multirow[t]{2}{*}{$H_{R / L}(6,1,1)_{422}$} & $H_{3 R / L}\left(3,1,1,-\frac{2}{3}\right)_{3221}$ & $H_{3 R / L}\left(3,1,-\frac{1}{3}\right)_{321}$ \\
\hline & $H_{\overline{3} R / L}\left(\overline{3}, 1,1, \frac{2}{3}\right)_{3221}$ & $H_{\overline{3} R / L}\left(\overline{3}, 1, \frac{1}{3}\right)_{321}$ \\
\hline \multirow[t]{4}{*}{$\Sigma(15,1,1)_{422}$} & $\Sigma_{1}(1,1,1,0)_{3221}$ & $\Sigma_{1}(1,1,0)_{321}$ \\
\hline & $\Sigma_{3}\left(3,1,1, \frac{4}{3}\right)_{3221}$ & $\Sigma_{3}\left(3,1, \frac{2}{3}\right)_{321}$ \\
\hline & $\Sigma_{\overline{3}}\left(\overline{3}, 1,1,-\frac{4}{3}\right)_{3221}$ & $\Sigma_{\overline{3}}\left(\overline{3}, 1,-\frac{2}{3}\right)_{321}$ \\
\hline & $\Sigma_{8}(8,1,1,0)_{3221}$ & $\Sigma_{8}(8,1,0)_{321}$ \\
\hline \multirow[t]{4}{*}{$\widetilde{\Sigma}(15,2,2)_{422}$} & $\widetilde{\Sigma}_{1}(1,2,2,0)_{3221}$ & $\widetilde{\Sigma}_{1}\left(1,2, \frac{1}{2}\right)_{321}, \widetilde{\Sigma}_{1}^{\prime}\left(1,2,-\frac{1}{2}\right)_{321}$ \\
\hline & $\widetilde{\Sigma}_{3}\left(3,2,2, \frac{4}{3}\right)_{3221}$ & $\widetilde{\Sigma}_{3}\left(3,2, \frac{7}{6}\right)_{321}, \widetilde{\Sigma}_{3}^{\prime}\left(3,2, \frac{1}{6}\right)_{321}$ \\
\hline & $\widetilde{\Sigma}_{\overline{3}}\left(\overline{3}, 2,2,-\frac{4}{3}\right)_{3221}$ & $\widetilde{\Sigma}_{\overline{3}}\left(\overline{3}, 2,-\frac{1}{6}\right)_{321}, \widetilde{\Sigma}_{\overline{3}}^{\prime}\left(\overline{3}, 2,-\frac{7}{6}\right)_{321}$ \\
\hline & $\widetilde{\Sigma}_{8}(8,2,2,0)_{3221}$ & $\widetilde{\Sigma}_{8}\left(8,2, \frac{1}{2}\right)_{321}, \widetilde{\Sigma}_{8}^{\prime}\left(8,2,-\frac{1}{2}\right)_{321}$ \\
\hline
\end{tabular}

Table 2. The decomposition of various $G_{422}$ representations into those of $G_{3221}$ and $G_{321}$ (SM). The table is from ref. [2] except signs and normalizations of the charges have been changed to conform to the more commonly used convention so that $Q_{\mathrm{em}}=I_{3}^{L}+Y=I_{3}^{L}+I_{3}^{R}+(B-L) / 2$. All the color triplet fields have quantum numbers corresponding to some leptoquark though that does not guarantee that they couple to lepton-quark pairs.

and we finally find the leptoquark we seek: a leptoquark $S_{1}=H_{3 L}^{*}\left(\overline{3}, 1, \frac{1}{3}\right)_{321}$ which couples to left-handed fermions. The field $H_{\overline{3} L}\left(\overline{3}, 1, \frac{1}{3}\right)_{321}$ has the same quantum numbers, but this one couples only to diquarks. Thus, we have surveyed the three NCG models and found a unique field, $H_{3 L}\left(3,1,-\frac{1}{3}\right)_{321}$ in Model C, which serves the purpose of the leptoquark $S_{1}\left(\overline{3}, 1, \frac{1}{3}\right)_{321}$ coupled to left-handed fermions.

\section{Gauge coupling unification}

In the following, we inquire whether the required unification of gauge couplings in the NCG models can be achieved. We focus specifically on Model C since it is the only model, among the NCG based Pati-Salam models, that contains the required leptoquark $H_{3 L}\left(3,1,-\frac{1}{3}\right)_{321}$. We find that in Model $\mathrm{C}$ with a light leptoquark $H_{3 L}\left(3,1,-\frac{1}{3}\right)_{321}$, it 
is necessary for at least one intermediate symmetry breaking scale to exist between the unification scale $M_{U}$, the scale at which the model emerges from an underlying NCG with a unified coupling, and the electroweak scale $M_{Z}$. The gauge couplings in these models do not unify otherwise. We consider several versions of the model with a single intermediate scale $M_{C}\left(M_{Z}<M_{C}<M_{U}\right)$ as illustrative examples. There, the symmetry $G_{422 D}$ that Model $\mathrm{C}$ possesses when it emerges at $M_{U}$ is assumed to persist down to $M_{C}$, at which point it breaks to the $G_{321}$ of the SM. Note that this specific sequence of symmetry breaking appears to be favoured by various phenomenological bounds [77].

\subsection{1-loop renormalization group running}

For a given particle content, the gauge couplings evolve under 1-loop renormalization group (RG) running in an energy interval $\left[M_{A}, M_{B}\right]$ following

$$
\frac{1}{g_{i}^{2}\left(M_{A}\right)}-\frac{1}{g_{i}^{2}\left(M_{B}\right)}=\frac{a_{i}}{8 \pi^{2}} \ln \frac{M_{B}}{M_{A}},
$$

where the RG coefficients $a_{i}$ are given by $[78,79]$

$$
\begin{aligned}
a_{i}= & -\frac{11}{3} C_{2}\left(G_{i}\right)+\frac{2}{3} \sum_{R_{f}} T_{i}\left(R_{f}\right) \cdot d_{1}\left(R_{f}\right) \cdots d_{n}\left(R_{f}\right) \\
& +\frac{\eta}{3} \sum_{R_{s}} T_{i}\left(R_{s}\right) \cdot d_{1}\left(R_{s}\right) \cdots d_{n}\left(R_{s}\right) .
\end{aligned}
$$

The summations in eq. (5.2) are, respectively, over irreducible chiral representations of fermions $\left(R_{f}\right)$ in the second term and those of scalars $\left(R_{s}\right)$ in the third. The coefficient $\eta$ is either 1 or $1 / 2$, depending on whether the corresponding representation is complex or (pseudo) real, respectively. $C_{2}\left(G_{i}\right)$ is the quadratic Casimir for the adjoint representation of the group $G_{i}$, and $T_{i}$ is the Dynkin index of each representation. For the U(1) group, $C_{2}(G)=0$ and

$$
\sum_{f, s} T=\sum_{f, s} Y^{2}
$$

where $Y$ is the $\mathrm{U}(1)$ charge. Particles that are heavier than $M_{B}$ (we assume $M_{A}<M_{B}$ ) decouple from the running and do not contribute to the sums in eq. (5.2). So the values of the RG coefficients $a_{i}$ change every time a symmetry breaking scale is crossed and some of the particles acquire masses of the order of that scale.

The low energy data which we will use as the boundary conditions to the RG running (in the $\overline{\mathrm{MS}}$ scheme) are $[80,81]$

$$
\begin{aligned}
\alpha^{-1}\left(M_{Z}\right) & =127.950 \pm 0.017, \\
\alpha_{s}\left(M_{Z}\right) & =0.1182 \pm 0.0016, \\
\sin ^{2} \theta_{W}\left(M_{Z}\right) & =0.23129 \pm 0.00005,
\end{aligned}
$$

at $M_{Z}=91.1876 \pm 0.0021 \mathrm{GeV}$, which translate to

$$
\begin{aligned}
& g_{1}\left(M_{Z}\right)=0.357419 \pm 0.000026, \\
& g_{2}\left(M_{Z}\right)=0.651765 \pm 0.000083, \\
& g_{3}\left(M_{Z}\right)=1.21875 \pm 0.00825 .
\end{aligned}
$$




\begin{tabular}{|cccc|}
\hline Representation & $\mathrm{SU}(2)$ & $\mathrm{SU}(3)$ & $\mathrm{SU}(4)$ \\
\hline \hline 2 & $\frac{1}{2}$ & - & - \\
3 & 2 & $\frac{1}{2}$ & - \\
4 & 5 & - & $\frac{1}{2}$ \\
6 & $\frac{35}{2}$ & $\frac{5}{2}$ & 1 \\
8 & 42 & 3 & - \\
10 & $\frac{165}{2}$ & $\frac{15}{2}$ & 3 \\
15 & 280 & $10, \frac{35}{2}$ & 4 \\
\hline
\end{tabular}

Table 3. Dynkin index $T_{i}$ for several irreducible representations of $\mathrm{SU}(2), \mathrm{SU}(3)$, and $\mathrm{SU}(4)$. Different normalization conventions are used in the literature. For instance, there is a factor of 2 difference between those given in ref. [79] and those in ref. [82]. Our convention follows the former. Notice that for $\mathrm{SU}(3)$, there exist two inequivalent 15 dimensional irreducible representations.

Throughout the RG running from the unification scale $M_{U}$ down to the electroweak scale $M_{Z}$, the coupling constants are all required to remain in the perturbative regime to justify the use of eq. (5.1).

\subsection{Does the leptoquark help coupling unification?}

We first illustrate that unification of the couplings cannot be realized when no intermediate symmetry breaking scales between $M_{U}$ and $M_{Z}$ are present. Since the symmetry just above $M_{Z}$ must be that of the SM, $G_{321}$, we are considering the situation in which the symmetry of Model C breaks immediately to $G_{321}$ at $M_{U}$, the scale at which the model emerges with a higher symmetry $G_{422 D}$ from the underlying NCG.

Thus the symmetry of the models in the interval $I=\left[M_{Z}, M_{U}\right]$ is $G_{321}$, and the vector and fermion content is just that of the SM. For the scalar content below $M_{U}$, we consider the fields coming from $\phi(1,2,2)_{422}$ and $H_{L}(6,1,1)_{422}$ :

$$
\begin{array}{lll}
\phi(1,2,2)_{422} & \rightarrow & \phi_{2}\left(1,2, \frac{1}{2}\right)_{321}+\phi_{2}^{\prime}\left(1,2,-\frac{1}{2}\right)_{321}, \\
H_{L}(6,1,1)_{422} & \rightarrow & H_{3 L}\left(3,1,-\frac{1}{3}\right)_{321}+H_{\overline{3} L}\left(\overline{3}, 1, \frac{1}{3}\right)_{321}
\end{array} .
$$

We identify $\phi_{2}\left(1,2, \frac{1}{2}\right)_{321}$ with the SM doublet, and $H_{3 L}\left(3,1,-\frac{1}{3}\right)_{321}$ is the scalar leptoquark necessary to explain the $B$-decay anomalies. We assume that the $H_{\overline{3} L}\left(\overline{3}, 1, \frac{1}{3}\right)_{321}$ field, which only couples to diquarks, becomes heavy when SU(4) breaks to $\mathrm{SU}(3)$ and does not survive to low energies. For the second Higgs doublet $\phi_{2}^{\prime}\left(1,2,-\frac{1}{2}\right)_{321}$, we have a choice of allowing it to survive to low energies after the breaking of $\mathrm{SU}(2)_{R}$, in which case we have a 2-Higgs doublet model (2HDM), or making it heavy and decoupling it after the breaking. 
As the scalar content, we will therefore consider the following two cases:

$$
\begin{array}{lll}
I_{1}: & \phi_{2}\left(1,2, \frac{1}{2}\right)_{321} \text { the SM Higgs, } H_{3 L}\left(3,1,-\frac{1}{3}\right)_{321} \\
I_{2}: & \phi_{2}\left(1,2, \frac{1}{2}\right)_{321} \text { the SM Higgs, } \phi_{2}^{\prime}\left(1,2,-\frac{1}{2}\right)_{321}, H_{3 L}\left(3,1,-\frac{1}{3}\right)_{321}
\end{array}
$$

The rest of the degrees of freedom are assumed to become heavy at the unification scale $M_{U}$. With this particle content, the RG coefficients are given by $a_{i}=a_{i}^{\mathrm{SM}}+\Delta a_{i},(i=1,2,3)$ where

$$
\begin{aligned}
a_{i}^{\mathrm{SM}} & =\left[\frac{41}{6},-\frac{19}{6},-7\right], \\
\Delta a_{i} & =\left[\frac{1}{9}\left(\frac{5}{18}\right), 0\left(\frac{1}{6}\right), \frac{1}{6}\left(\frac{1}{6}\right)\right],
\end{aligned}
$$

for model $I_{1}\left(I_{2}\right)$.

The symmetry of the model $G_{442 D}$ is broken to the symmetry of the SM $G_{321}$ at $M_{U}$, which is also the scale where it emerges from an underlying NCG. The obvious boundary/matching conditions to be imposed on the couplings at $M_{U}$ and $M_{Z}$ are:

$$
\begin{aligned}
& M_{U}: \sqrt{\frac{5}{3}} g_{1}\left(M_{U}\right)=g_{2}\left(M_{U}\right)=g_{3}\left(M_{U}\right), \\
& M_{Z}: \frac{1}{e^{2}\left(M_{Z}\right)}=\frac{1}{g_{1}^{2}\left(M_{Z}\right)}+\frac{1}{g_{2}^{2}\left(M_{Z}\right)} .
\end{aligned}
$$

Using eq. (5.1) with the low energy data and boundary conditions given in eqs. (5.4) and (5.9), the following equations are obtained.

$$
\begin{aligned}
2 \pi\left[\frac{3-8 \sin ^{2} \theta_{w}\left(M_{Z}\right)}{\alpha\left(M_{Z}\right)}\right] & =\left(3 a_{1}-5 a_{2}\right) \ln \frac{M_{U}}{M_{Z}}, \\
2 \pi\left[\frac{3}{\alpha\left(M_{Z}\right)}-\frac{8}{\alpha_{s}\left(M_{Z}\right)}\right] & =\left(3 a_{1}+3 a_{2}-8 a_{3}\right) \ln \frac{M_{U}}{M_{Z}} .
\end{aligned}
$$

Taking the ratio of these equations, which also cancels the uncertainty on $M_{Z}$, yields the condition to be satisfied for unification as

$$
r \equiv \frac{3 a_{1}-5 a_{2}}{3 a_{1}+3 a_{2}-8 a_{3}} \cong 0.4656 \pm 0.0014
$$

Using eq. (5.8) we find that the models $I_{1}$ and $I_{2}$ yield $r_{1} \cong 0.56$ and $r_{2} \cong 0.54$, respectively. Neither of these values fall in the required range stated above. The latter value, $r_{2}$, is actually identical to $r_{\mathrm{SM}}$ by coincidence; the modifications in both the numerator and denominator of $r_{2}$ cancel. Thus, allowing the second Higgs doublet $\phi_{2}^{\prime}\left(1,2, \frac{1}{2}\right)_{321}$ to survive in model $I_{2}$ does not help in unifying the couplings.

The resulting non-unification of the couplings can also be observed in figure 4, where their RG runnings are displayed. Notice that the overall running of the couplings in Model $I_{1}$ and Model $I_{2}$ are almost identical to the one in the SM due to the smallness of the ratios $\Delta a_{i} / a_{i}^{\mathrm{SM}}$. Furthermore, in the case of Model $I_{2}$, the three intersection points (of any 


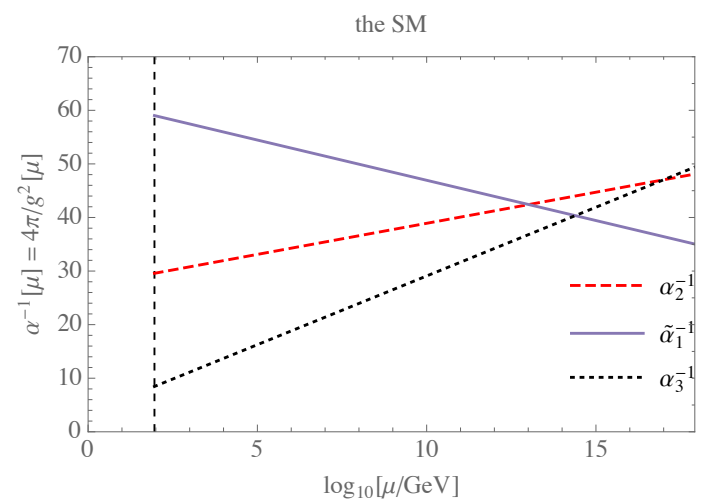

(a)

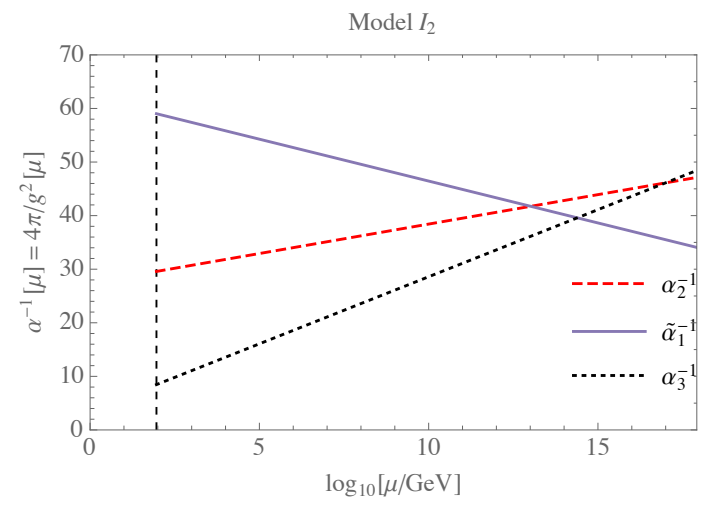

(c)

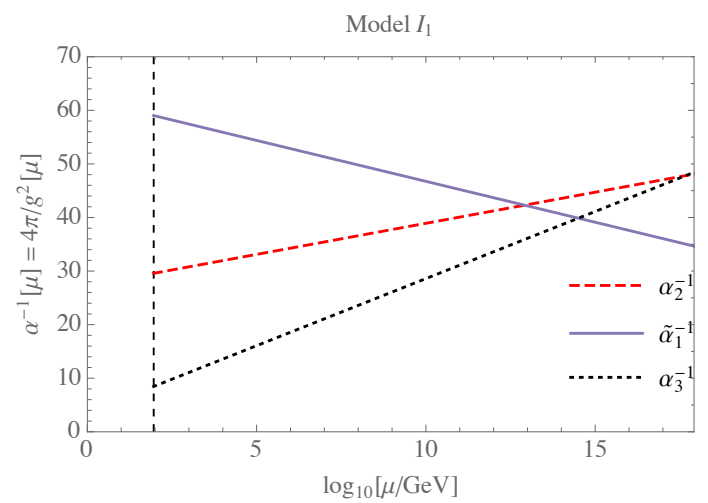

(b)

Figure 4. Running of the gauge couplings for the SM, Model $I_{1}$, and Model $I_{2}$. The vertical dotted line correspond to the electroweak scale $M_{Z}$. For $\alpha_{1}^{-1}$, we plot the redefined quantity $\widetilde{\alpha}_{1}^{-1} \equiv \frac{3}{5} \alpha_{1}^{-1}$. Including the leptoquark with or without a second Higgs doublet does not make a significant modification to the SM running in favor of unification. Therefore in the NCG framework, one needs to consider intermediate symmetry breaking scales for these options. Note that the running of the couplings in the case of the SM and Model $I_{2}$ is almost identical due to accidental cancellations of additional contributions in the 1-loop RG coefficients $\left(\Delta a_{i}\right)$ of Model $I_{2}$.

pair of couplings out of the three), which are controlled by the combinations $\left(3 a_{1}-5 a_{2}\right)$, $\left(3 a_{1}-5 a_{3}\right)$, and $\left(a_{2}-a_{3}\right)$, respectively, occur at the same scales as in the SM running because the extra parts in these combinations, coming from $\Delta a_{i}$, cancel in the case of Model $I_{2}$. Although the intersection scales are the same between Model $I_{2}$ and the SM, the overall evolution of the couplings is still slightly different since each $\Delta a_{i}$ is nonzero in Model $I_{2}$. The modification manifests itself in the values of $\alpha_{i}^{-1}$ at each intersection point. This is obviously an accident in the 1-loop running. Going to the 2-loop level would most likely cause non-zero contributions in the relevant combinations $a_{i}$, but these can be expected to be extremely suppressed and hence still would not provide an overall coupling unification. 
So far, we have assumed that the leptoquark $H_{3 L}\left(3,1,-\frac{1}{3}\right)_{321}$ is at the TeV-scale and that its companion $H_{\overline{3} L}\left(\overline{3}, 1, \frac{1}{3}\right)_{321}$, the other color-triplet contained in $H_{L}(6,1,1)_{422}$, is heavy at the unification scale $M_{U}$, where the Pati-Salam structure emerges from the underlying NCG, and where the Pati-Salam symmetry, $G_{422 D}$, breaks into the symmetry of the SM, as well. We have considered two versions of this scenario depending on whether the second Higgs doublet $\phi_{2}^{\prime}\left(1,2,-\frac{1}{2}\right)_{321}$, contained in $\phi(1,2,2)_{422}$ together with the SM Higgs doublet $\phi_{2}\left(1,2, \frac{1}{2}\right)_{321}$, is light at the TeV-scale or heavy at the unification scale. We have shown that the running of the couplings is not improved in either of these cases.

Now, we will try a relatively general strategy to look for any improvement in favor of coupling unification. Instead of assuming that the second leptoquark, $H_{\overline{3} L}\left(\overline{3}, 1, \frac{1}{3}\right)_{321}$, and the second Higgs doublet $\phi_{2}^{\prime}\left(1,2,-\frac{1}{2}\right)_{321}$ are either at the TeV-scale or the unification scale, we will let their masses float in between these scales in order to search for intervals of mass values leading to direct coupling unification. Note that we still assume at this stage that there is no intermediate symmetry breaking. Therefore, the mass values of these particles are not dictated by a symmetry breaking mechanism but possibly by an another, yet to be determined, mechanism or they are picked randomly by the model by sheer accident. Note that we still keep the mass of our main leptoquark $H_{3 L}\left(3,1,-\frac{1}{3}\right)_{321}$ at the TeV scale, required by the possible explanation for the $B$-decay anomalies. Then, eq. (5.10) changes in the following way: ${ }^{13}$

$$
\begin{aligned}
& 2 \pi\left[\frac{3-8 \sin ^{2} \theta_{w}\left(M_{Z}\right)}{\alpha\left(M_{Z}\right)}\right] \\
& =\left(3 a_{1}-5 a_{2}\right)^{\mathrm{I}} \ln \frac{M_{X}}{M_{Z}}+\left(3 a_{1}-5 a_{2}\right)^{\mathrm{II}} \ln \frac{M_{Y}}{M_{X}}+\left(3 a_{1}-5 a_{2}\right)^{\mathrm{III}} \ln \frac{M_{U}}{M_{Y}}, \\
& 2 \pi\left[\frac{3}{\alpha\left(M_{Z}\right)}-\frac{8}{\alpha_{s}\left(M_{Z}\right)}\right] \\
& =\left(3 a_{1}+3 a_{2}-8 a_{3}\right)^{\mathrm{I}} \ln \frac{M_{X}}{M_{Z}}+\left(3 a_{1}+3 a_{2}-8 a_{3}\right)^{\mathrm{II}} \ln \frac{M_{Y}}{M_{X}}+\left(3 a_{1}+3 a_{2}-8 a_{3}\right)^{\mathrm{III}} \ln \frac{M_{U}}{M_{Y}},
\end{aligned}
$$

where $M_{X}$ and $M_{Y}$ denote, respectively, the lighter and heavier mass among the second Higgs doublet and the second leptoquark. Let us first consider the case where the second Higgs doublet is heavier, which we call model $T_{1}$ (and $T_{2}$ for the opposite case). Then, we have $M_{Y} \equiv M_{\phi_{2}^{\prime}}$ and $M_{X} \equiv M_{H_{\overline{3} L}}$. Therefore, in the fist interval (I) the active degrees of freedom, in addition to the SM particles, come only from our TeV-scale

\footnotetext{
${ }^{13}$ The threshold corrections can be safely ignored throughout this paper. The ignored terms are in the form of $-\Delta f_{c}\left(a_{i}\right) \ln \left(1-\epsilon_{c}\right)$, where $\epsilon_{c} \equiv \frac{M_{c}-M_{\delta_{c}}}{M_{c}}$ and $c=X, Y, U$, labelling each term according to the larger mass in each logarithm in eq. (5.12). $\Delta f_{c}\left(a_{i}\right)$ are the difference between the values of corresponding combinations of $a_{i}$ in front of each logarithmic factor, above and below the threshold scale $M_{\delta_{c}}$, respectively. For instance, the correction term for the first term in the first equation in eq. (5.12) becomes $\left[\left(3 a_{1}-5 a_{2}\right)^{\mathrm{II}}-\left(3 a_{1}-5 a_{2}\right)^{\mathrm{I}}\right] \ln \frac{M_{X}}{M_{\delta_{X}}}$. From the effective field theory point of view, these terms are expected to become relevant as $M_{\delta_{c}} \rightarrow M_{c}$, or $\epsilon_{c} \rightarrow 0$, but then they are suppressed by a factor proportional $O\left(\epsilon_{c}\right)$ due to the natural logarithm, unless $\Delta f_{c}\left(a_{i}\right)$ is enormous which is rarely the case in general, and definitely not the case for the scenarios considered in this paper.
} 


\begin{tabular}{|c|l|l|}
\hline Interval & $\begin{array}{l}\text { active scalar dofs for } \\
\text { model } T_{1}\left(T_{2}\right)\end{array}$ & RG coefficients \\
\hline III $\left(M_{U}-M_{Y}\right)$ & $\phi_{2}, H_{3 L}, H_{\overline{3} L}, \phi_{2}^{\prime}$ & {$\left[a_{1}, a_{2}, a_{3}\right]^{\mathrm{III}}=\left[\frac{65}{9},-3,-\frac{20}{3}\right]$} \\
\hline II $\left(M_{Y}-M_{X}\right)$ & $\phi_{2}, H_{3 L}, H_{\overline{3} L}$ & {$\left[a_{1}, a_{2}, a_{3}\right]^{\mathrm{II}}$} \\
& $\left(\phi_{2}, H_{3 L}, \phi_{2}^{\prime}\right)$ & $=\left[\frac{127}{18}\left(\frac{64}{9}\right),-\frac{19}{6}(-3),-\frac{20}{3}\left(-\frac{41}{6}\right)\right]$ \\
\hline I $\left(M_{X}-M_{Z}\right)$ & $\phi_{2}, H_{3 L}$ & {$\left[a_{1}, a_{2}, a_{3}\right]^{\mathrm{I}}=\left[\frac{125}{18},-\frac{19}{6},-\frac{41}{6}\right]$} \\
\hline
\end{tabular}

Table 4. The Higgs content and the RG coefficients in the energy intervals for model $T_{1,2}$. Recall that all the fields above are complex.

leptoquark $H_{3 L}\left(3,1,-\frac{1}{3}\right)_{321}$. In the second interval (II) we add in the second leptoquark, $H_{\overline{3} L}\left(\overline{3}, 1, \frac{1}{3}\right)_{321}$, and finally in the third interval (III), all of the degrees of freedom are active.

Using the RG coefficients given in table 4, eq. (5.12) becomes

$$
\begin{aligned}
& 3270=110 u+y-x, \\
& 2283=66 u-y+x,
\end{aligned}
$$

where

$$
u \equiv \ln \frac{M_{U}}{\mathrm{GeV}}, \quad y \equiv \ln \frac{M_{Y}}{\mathrm{GeV}}, \quad x \equiv \ln \frac{M_{X}}{\mathrm{GeV}} .
$$

Solving these equations yields

$$
u=31.55, \quad y=x-200.63
$$

which clearly violates our necessary condition that $u \geqslant y \geqslant x$ and hence, does not constitute a solution for our system. Similarly for model $T_{2}$, at which the second leptoquark is heavier than the second Higgs doublet, i.e. $M_{Y} \equiv M_{H_{\overline{3}}}$ and $M_{X} \equiv \phi_{2}^{\prime}$, eq. (5.12) reads

$$
\begin{aligned}
& 3270=110 u-y+x \\
& 2283=66 u+y-x,
\end{aligned}
$$

which yields

$$
u=31.55, \quad y=x+200.63,
$$

violating again the condition $u \geqslant y \geqslant x$, and thus, no acceptable solution exists in this case as well.

Therefore, we conclude that unification of the couplings cannot be directly realized in these scenarios. There should exist at least one intermediate symmetry breaking phase between the unification scale $M_{U}$ and the weak scale, as we illustrate in the next section. 
However, we would like to note that we adopt here quite a minimalistic approach. If one becomes willing to include more degrees of freedom in the light(er) spectrum, it is quite likely that unification of the gauge couplings is realized with no intermediate symmetry breaking $[33,34]$.

\subsection{Unification with a single intermediate scale}

Next, we demonstrate that the unification of the couplings in Model $\mathrm{C}$ with a light leptoquark $H_{3 L}\left(3,1,-\frac{1}{3}\right)_{321} \equiv S_{1}^{*}$ can be realized with the introduction of a single intermediate symmetry breaking step. According to this scenario, the Pati-Salam symmetric phase is intact from the unification scale $M_{U}$, where the Pati-Salam structure emerges from a noncommutative geometry, down to the intermediate energy scale $M_{C}\left(M_{Z}<M_{C}<M_{U}\right)$, at which the $G_{422 D}$ symmetry spontaneously breaks down to $G_{321}$ of the SM.

In Model $\mathrm{C}$, the main field surviving down to low energies in addition to the SM fields is the leptoquark $H_{3 L}\left(3,1,-\frac{1}{3}\right)_{321}$, originated from the complex field $H_{L}(6,1,1)_{422}$. The other leptoquark, $H_{\overline{3} L}\left(\overline{3}, 1, \frac{1}{3}\right)_{321}$, also contained in $H_{L}(6,1,1)_{422}$, is assumed to be heavy at the scale $M_{C}$. We consider two versions of this model, we call model $C_{1}$ and model $C_{2}$, depending on the difference based on whether the second scalar doublet, contained in the complex $\phi(1,2,2)_{422}$, remains heavy at the scale $M_{C}$, or it becomes light and survives down to the low energies with the other scalar doublet of the same Pati-Salam multiplet, the SM Higgs. In the latter case, the low energy section of the model can be parameterized as the $2 \mathrm{HDM}$, augmented by the leptoquark $H_{3 L}\left(3,1,-\frac{1}{3}\right)_{321} \cdot{ }^{14}$

Therefore, the scalar content of the models at the TeV-scale are the same as in eq. (5.7), while, here, we have a different sequence of symmetry breaking. The symmetry breaking chain we consider here has been discussed in detail in our previous papers $[1,2]$ :

$$
\mathrm{NCG} \stackrel{M_{U}}{\longrightarrow} G_{422 D} \underset{\left\langle\Delta_{R}\right\rangle}{\stackrel{M_{C}}{\longrightarrow}} G_{321},
$$

where the double arrow points to the symmetry emerging from the underlying NCG, while the single arrow denotes the spontaneous symmetry breaking in the usual way.

We label the energy intervals in between symmetry breaking scales $\left[M_{Z}, M_{C}\right]$ and $\left[M_{C}, M_{U}\right]$ with Roman numerals as

$$
\begin{array}{rll}
\text { I }: & {\left[M_{Z}, M_{C}\right],} & G_{321}(\mathrm{SM}), \\
\text { II }: & {\left[M_{C}, M_{U}\right],} & G_{422 D} .
\end{array}
$$

The generic boundary/matching conditions to be imposed on the couplings at the symmetry breaking scales are given as:

$$
\begin{array}{rlrl}
M_{U}: & g_{L}\left(M_{U}\right)=g_{R}\left(M_{U}\right)=g_{4}\left(M_{U}\right), & \\
M_{C}: & g_{3}\left(M_{C}\right)=g_{4}\left(M_{C}\right), & g_{2}\left(M_{C}\right)=g_{L}\left(M_{C}\right), \\
& \frac{1}{g_{1}^{2}\left(M_{C}\right)}=\frac{1}{g_{R}^{2}\left(M_{C}\right)}+\frac{2}{3} \frac{1}{g_{4}^{2}\left(M_{C}\right)}, & & g_{L}\left(M_{C}\right)=g_{R}\left(M_{C}\right), \\
M_{Z}: & \frac{1}{e^{2}\left(M_{Z}\right)}=\frac{1}{g_{1}^{2}\left(M_{Z}\right)}+\frac{1}{g_{2}^{2}\left(M_{Z}\right)} . &
\end{array}
$$

\footnotetext{
${ }^{14} \mathrm{~A}$ study of $\mathrm{SO}(10)$ realization of such a model and its LHC phenomenology is currently under preparation by Aydemir et al. .
} 


\begin{tabular}{|c|l|l|}
\hline Interval & Higgs content for model $C_{1}\left(C_{2}\right)$ & RG coefficients \\
\hline II & $\phi(1,2,2)_{422}, \widetilde{\Sigma}(15,2,2)_{422}$, & {$\left[a_{L}, a_{R}, a_{4}\right]^{\mathrm{II}}=\left[\frac{26}{3}, \frac{26}{3}, \frac{4}{3}\right]$} \\
& $\Delta_{R}(10,1,3)_{422}, H_{R}(6,1,1)_{422}$ & \\
\hline I & $\Delta_{L}(10,3,1)_{422}, H_{L}(6,1,1)_{422}$ & \\
& $\phi_{2}\left(1,2, \frac{1}{2}\right)_{321}\left(+\phi_{2}^{\prime}\left(1,2,-\frac{1}{2}\right)_{321}\right.$ for $\left.C_{2}\right)$, & {$\left[a_{1}, a_{2}, a_{3}\right]^{\mathrm{I}}$} \\
& $H_{3 L}\left(3,1,-\frac{1}{3}\right)_{321}$ & {$\left[\frac{125}{18}\left(\frac{64}{9}\right),-\frac{19}{6}(-3),-\frac{41}{6}\right]$} \\
\hline
\end{tabular}

Table 5. The Higgs content and the RG coefficients in the energy intervals for model $C_{1,2}$. Recall that all the fields above are complex.

\begin{tabular}{|c||c|c|}
\hline Model & $C_{1}$ & $C_{2}$ \\
\hline \hline UV Symmetry & $G_{422 D}$ & $G_{422 D}$ \\
\hline $\log _{10}\left(M_{U} / \mathrm{GeV}\right)$ & 15.7 & 15.4 \\
$\log _{10}\left(M_{C} / \mathrm{GeV}\right)$ & 13.7 & 13.7 \\
\hline$\alpha_{U}^{-1}$ & 36.9 & 37.0 \\
\hline
\end{tabular}

Table 6. The predictions of models $C_{1}$ and $C_{2}$.

Using eq. (5.1) with the boundary conditions given in eqs. (5.4) and (5.20), one obtains the relevant equations as the following.

$$
\begin{aligned}
& 2 \pi\left[\frac{3-8 \sin ^{2} \theta_{W}\left(M_{Z}\right)}{\alpha\left(M_{Z}\right)}\right]=\left(3 a_{1}-5 a_{2}\right)^{\mathrm{I}} \ln \frac{M_{C}}{M_{Z}}+\left(-5 a_{L}+3 a_{R}+2 a_{4}\right)^{\mathrm{II}} \ln \frac{M_{U}}{M_{C}}, \\
& 2 \pi\left[\frac{3}{\alpha\left(M_{Z}\right)}-\frac{8}{\alpha_{s}\left(M_{Z}\right)}\right]=\left(3 a_{1}+3 a_{2}-8 a_{3}\right)^{\mathrm{I}} \ln \frac{M_{C}}{M_{Z}}+\left(3 a_{L}+3 a_{R}-6 a_{4}\right)^{\mathrm{II}} \ln \frac{M_{U}}{M_{C}},
\end{aligned}
$$

where the low energy input is collected on the left hand side. The corresponding beta coefficients for each interval are given in table 5. Additionally, the unified coupling $\alpha_{U}$ at scale $M_{U}$ can be obtained from

$$
\frac{2 \pi}{\alpha_{U}}=\frac{2 \pi}{\alpha_{s}\left(M_{Z}\right)}-\left(a_{4}^{\mathrm{II}} \ln \frac{M_{U}}{M_{C}}+a_{3}^{\mathrm{I}} \ln \frac{M_{C}}{M_{Z}}\right) .
$$

The results are given in table 6 and in figure 5, where in the latter the unification of the couplings is displayed. The difference between two models is minor, as expected. In both models, the unification scales are far away from the Planck scale so that we can safely ignore gravitational effects. Moreover, the value of intermediate symmetry breaking scale, $M_{C} \simeq 10^{13.7} \mathrm{GeV}$, which is almost the same in both models, is consistent with the current bounds coming from the proton decay searches. Since this is the scale at which the 


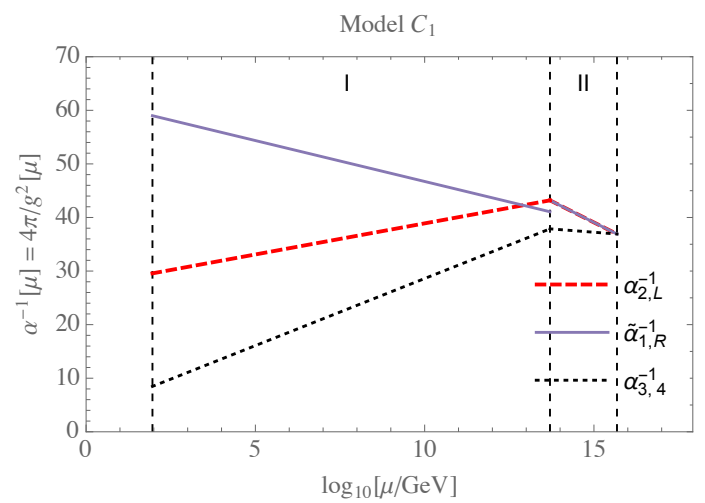

(a)

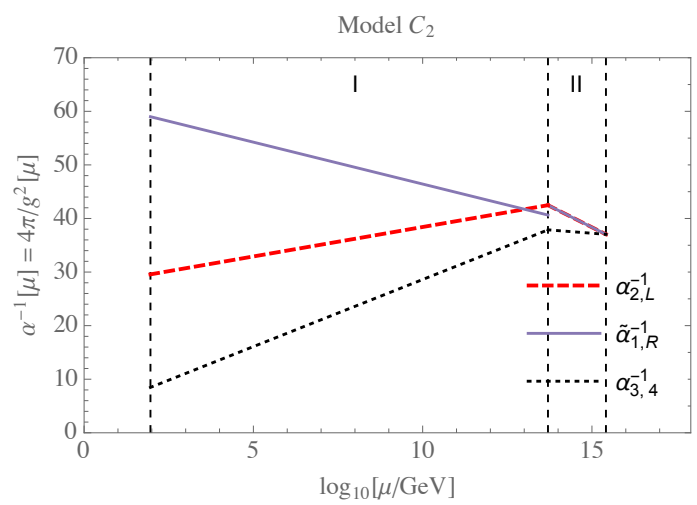

(b)

Figure 5. Running of the gauge couplings for models $C_{1}$, and $C_{2}$. The vertical dotted lines correspond to the electroweak scale $M_{Z}, M_{C}$, and the unification scale $M_{U}$. Note again that $\widetilde{\alpha}_{1}^{-1} \equiv \frac{3}{5} \alpha_{1}^{-1}$

Pati-Salam symmetry beaks into the SM, it determines the expected mass values for the proton-decay-mediating leptoquarks. From a naive analysis [77], it can be shown that the current bounds on the proton lifetime $[80,83]$ requires $M_{C} \gtrsim 10^{11} \mathrm{GeV}$.

We discuss the proton stability more in the next, concluding, section, in which we address also some conceptual issues related to the NCG approach to the SM and its natural Pati-Salam-like completion.

\section{Discussion and conclusions}

\subsection{Discussion}

Motivated by scalar-leptoquark explanations of the recently reported $B$-decay anomalies, in this paper we have investigated whether the required leptoquarks can be accommodated within unified models based on NCG. As already pointed out, such NCG-based models have the gauge structure of Pati-Salam models, $\mathrm{SU}(4) \times \mathrm{SU}(2)_{L} \times \mathrm{SU}(2)_{R}$, with gauge coupling unification at a single scale. In one of the models we did find a unique scalar leptoquark $H_{3 L}\left(3,1,-\frac{1}{3}\right)_{321}$, originating from the multiplet $H_{L}(6,1,1)_{422}$, which can potentially explain the $B$-decay anomalies, following the phenomenological analysis of [26], provided its mass is on the order of a few TeV. Also, the unification of couplings can be realized with the inclusion of a single step of intermediate symmetry breaking. Note that $H_{L}(6,1,1)_{422}$ is complex and decomposes into $S_{1}^{*}\left(3,1,-\frac{1}{3}\right)_{321}$ and another field which couples only to diquarks. In order to avoid proton decay, we suppress the mixing between these two scalars. Furthermore, we assume that the field that couples only to diquarks is heavy to avoid any other phenomenological consequences. These assumptions might raise the question of naturalness, which is not different from the corresponding situation found in generic GUT models. As explained in the discussion that follows, it is an interesting feature of the NCG models that one obtains relatively light leptoquarks without obvious problems associated with proton decay. 
Given the restrictive nature of the unified Pati-Salam models in the NCG framework, we find the possible scalar leptoquark $S_{1}^{*}\left(3,1,-\frac{1}{3}\right)_{321}$ explanation of the $B$-decay anomalies particularly interesting. In particular, as pointed out in [26], this leptoquark can be used to explain not only the violation of lepton universality in $B$-decays and the enhanced rates in certain $B$-decays, but also the anomalous magnetic moment of the muon.

Note that a state with quantum numbers $\left(3,1,-\frac{1}{3}\right)_{321}$ exists in the 5 dimensional representation of $\mathrm{SU}(5)$ as well, and therefore it could provide the required phenomenological features. However, one light $\left(3,1,-\frac{1}{3}\right)_{321}$ state found in a non-supersymmetric framework, does not imply unification of couplings as the SU(5) GUT theory would require. The $\mathrm{SO}(10)$ picture, on the other hand, can accommodate this in a reasonable scenario (as in refs. [73, 84]) by including intermediate symmetry breaking scales.

Similarly, unification of couplings in the NCG models could be realized by imposing (at least) one intermediate scale. According to the scenario we considered in this paper, the Pati-Salam symmetry, $G_{422}=\mathrm{SU}(4)_{C} \otimes \mathrm{SU}(2)_{L} \otimes \mathrm{SU}(2)_{R}$, emerges from an underlying noncommutative geometry at a "unification" scale $M_{U}$, and stays intact down to the scale $M_{C}$, where it is spontaneously broken to the symmetry of the SM. As discussed in the section 4 of this paper, there are three different Pati-Salam models in the NCG framework $[3,4]$ depending on their scalar contents. However, only the $H_{L}(6,1,1)_{422}$ in Model $\mathrm{C}$ has the right coupling to left-handed fermions and it leads to the phenomenologically preferred leptoquark. And, as we discuss in the following subsection, the usual problems associated with proton decay can be avoided.

\subsection{Light colored scalars and proton decay}

Proton decay is not an issue of concern in our scenario, although we have a light scalar leptoquark $H_{3 L}\left(3,1,-\frac{1}{3}\right)_{321}$ (generally labeled as $S_{1}^{*}$ in the literature) in Model C, which possesses the right quantum numbers for it to couple to potentially dangerous diquark operators. The proton stability is ensured because the underlying non-commutative geometry does not allow these couplings to appear.

Oftentimes, the possible existence of light leptoquarks is dismissed, perhaps a bit too quickly, since they can potentially lead to proton decay. Although the operators contributing to proton decay should be carefully treated or possibly turned off in order not to contradict the experimental evidence, the underlying mechanism responsible for possible elimination of these operators may not be obvious at first glance. We see an example of this in Model C. In this model, the leptoquarks $H_{3 R / L}\left(3,1,-\frac{1}{3}\right)_{321}$ can safely be light since their diquark couplings are turned off by the underlying noncommutative geometry and hence do not contribute to proton decay; their companions, on the other hand, $H_{\overline{3} R / L}\left(\overline{3}, 1, \frac{1}{3}\right)_{321}$ (à la the parent-multiplets $\left.H_{R / L}(6,1,1)_{422}\right)$, possess these couplings, and therefore the mixing between them should be small. However, by looking only at the low energy behaviour of the model, from bottom-up perspective, one observes only the absence of the corresponding diquark couplings. From this viewpoint, there is no indication regarding the nature of the underlying mechanism, which is the non-commutative framework in this particular case.

In general, the ordinary, non-unified, Pati-Salam-type models, have the reputation of being safe in terms of proton stability. Although this is indeed the case in terms of the 
gauge-boson-mediated contributions, the situation is not so trivial for the colored-scalarmediated contributions; not every scalar sector choice guarantees the apparent stability of the proton. The most commonly chosen scalar sector in the ordinary Pati-Salam framework accommodates a global symmetry that prevents proton decay. This scalar sector consists of $\Delta_{R}(10,1,3)_{422}$ and $\Delta_{L}(10,3,1)_{422}$, in addition to the bidoublet $\phi(1,2,2)_{422}$. This is, at first glance, very similar to the scalar sector of Model C. However, this mechanism is due to the totally-symmetric nature of $\Delta_{L, R}$ under SU(4), and existence of any field(s) transforming anti-symmetrically would potentially spoil this symmetry [85]. In fact, this is exactly what we have in Model C; the multiplets $H_{R / L}(6,1,1)_{422}$ reside in the totallyanti-symmetric representations of SU(4). Fortunately enough, the restrictive nature of the non-commutative-geometry framework happens to operate in our favor and does not allow the proton-decay-inducing diquark couplings for our leptoquark $H_{3 L}\left(3,1,-\frac{1}{3}\right)_{321}$, thus offering a plausible explanation for the $B$-decay anomalies.

\subsection{NCG's pros and cons}

In this paper we have discussed yet another phenomenological aspect of the hidden noncommutative structure behind the Standard Model (and its natural Pati-Salam-like completion), that has been uncovered by Connes, Chamseddine and collaborators [8, 86]. Even though such non-commutative framework has been pointed out for some time, in light of the LHC's discovery of the Higgs boson, and the fact that the NCG of the SM is precisely relevant for the SM with the Higgs boson, we were motivated to explore other phenomenological implications of such a non-commutative approach $[1,2,76,87]$.

Note that the NCG of the SM might appear peculiar from the canonical effective field theory point of view, because of the way the SM action is rewritten, in a Fujikawa-like form familiar from the study of chiral anomalies in gauge theories, which introduces a scale, not a Wilsonian cut-off, and which in turn is fixed by the existence of one overall coupling, thus implying the unification of the three SM couplings at a GUT scale without GUT degrees of freedom. Furthermore, the NCG scheme hides a left-right symmetric structure which can be naturally broken to the canonical SM. Note that this is also an unusual left-right completion. First, this non-commutative completion has a GUT-like unification (like the NCG of the SM), which simply does not exist for the canonical leftright symmetric models. Second, this non-commutative left-right completion of the SM is quite constrained compared to the already existing effective field theory literature on the subject. As emphasized in the present paper, such unified Pati-Salam structure can lead to a unique scalar leptoquark which may potentially explain the $B$-decay anomalies, provided its mass is around a few $\mathrm{TeV}$, without causing any problems with proton decay.

One might wonder whether the NCG of the SM and the unified Pati-Salam models can have a reasonable UV completion. In particular, where could the NCG originate from a UV point of view? Here, in conclusion, we offer a few comments on this important question. We note that if one views quantum gravity coupled to Standard Model-like matter as having origins in string theory, then one can convincingly argue for an intrinsic non-commutative structure in that context [88-94]. This work also suggests that the low energy theory (such as the one described by the Standard Model and its Pati-Salam-like completion) is 
intrinsically non-commutative. In order to obtain the NCG of the SM and its Pati-Salam completion, from such a UV point of view, what is needed is just some suitable fermionic degrees of freedom, propagating in a background of induced gravitational and gauge degrees of freedom (of the SM or Pati-Salam type). Then after integrating over the fermionic zero modes the induced action of the Fujikawa form, needed by the NCG structure of the SM, naturally emerges. Note that the usual discussion of hierarchy of scales as well as the issue of decoupling of scales is different in this framework, which in principle allows for mixing between short distance and long distance degrees of freedom, thus shedding new light on the questions of naturalness and the hierarchy problem.

\section{Acknowledgments}

We thank Chris Hill, Paul Oehlmann, Nobuchika Okada, Leo Piilonen and Mark Pitt for discussions and Walter D. van Suijlekom for useful communications. UA is supported by the National Natural Science Foundation of China (NSFC) under Grant No. 11505067. DM thanks the Julian Schwinger Foundation for support. CS is supported in part by the International Postdoctoral Fellowship funded by China Postdoctoral Science Foundation, and is grateful for the support from JiJi Fan, Marcelo Gleiser, and Devin Walker.

\section{A Leptoquarks}

In this appendix we review the derivation of the most generic Lagrangian for scalar and vector leptoquark interactions with Standard Model (SM) fermions. We follow the notation of ref. [58], which updates ref. [59] with the inclusion of leptoquarks and interactions that involve the right-handed neutrino. All quantum number assignments refer to those in $G_{321}=\mathrm{SU}(3)_{C} \times \mathrm{SU}(2)_{L} \times \mathrm{U}(1)_{Y}$ of the SM.

At low-energies, we assume the fermionic content of the SM plus right-handed neutrinos:

$$
\begin{aligned}
Q_{L}^{i}=\left(3,2,+\frac{1}{6}\right), & d_{R}^{i}=\left(3,1,-\frac{1}{3}\right), & u_{R}^{i}=\left(3,1,+\frac{2}{3}\right), \\
L_{L}^{i}=\left(1,2,-\frac{1}{2}\right), & e_{R}^{i}=(1,1,-1), & \nu_{R}^{i}=(1,1,0) .
\end{aligned}
$$

where $i=1,2,3$ is the generation index. From this set of fermions, let us construct the most general quark-lepton bilinears. First, the scalars with fermion number $F=3 B+L=0$ are

$$
\begin{array}{ll}
\overline{u_{R}^{i}} L_{L}^{j}=\left(\overline{3}, 2,-\frac{7}{6}\right), & \overline{d_{R}^{i}} L_{L}^{j}=\left(\overline{3}, 2,-\frac{1}{6}\right), \\
\overline{Q_{L}^{i}} e_{R}^{j}=\left(\overline{3}, 2,-\frac{7}{6}\right), & \overline{Q_{L}^{i}} \nu_{R}^{j}=\left(\overline{3}, 2,-\frac{1}{6}\right),
\end{array}
$$

and their hermitian conjugates. Note that the bilinears in the same column above share the same $\mathrm{SU}(3)_{C} \times \mathrm{SU}(2)_{L} \times \mathrm{U}(1)_{Y}$ quantum numbers. The scalars with fermion number 


$$
\begin{aligned}
& F=2 \text { are } \\
& \begin{array}{lll}
\overline{Q_{L}^{C i}} \epsilon L_{L}^{j}=\left(3,1,-\frac{1}{3}\right), & \overline{u_{R}^{C i}} e_{R}^{j}=\left(3,1,-\frac{1}{3}\right), & \overline{d_{R}^{C i}} \nu_{R}^{j}=\left(3,1,-\frac{1}{3}\right), \\
\overline{Q_{L}^{C i}} \epsilon \vec{\tau} L_{L}^{j}=\left(3,3,-\frac{1}{3}\right), & \overline{d_{R}^{C i}} e_{R}^{j}=\left(3,1,-\frac{4}{3}\right), & \overline{u_{R}^{C i}} \nu_{R}^{j}=\left(3,1,+\frac{2}{3}\right),
\end{array}
\end{aligned}
$$

and their hermitian conjugates, where $\psi^{C}=C \bar{\psi}^{\mathrm{T}}$, and $\epsilon=i \tau_{2}$ in $\mathrm{SU}(2)_{L}$ isospin space which contracts the isospin indices of two isospinors. $\vec{\tau}=\left(\tau_{1}, \tau_{2}, \tau_{3}\right)$ are the Pauli matrices. Note that the bilinears in the first row above share the same quantum numbers. The $F=0$ vectors are

$$
\begin{array}{rlrl}
\overline{Q_{L}^{i}} \gamma^{\mu} L_{L}^{j} & =\left(\overline{3}, 1,-\frac{2}{3}\right), & \overline{d_{R}^{i}} \gamma^{\mu} e_{R}^{j}=\left(\overline{3}, 1,-\frac{2}{3}\right), & \overline{u_{R}^{i}} \gamma^{\mu} \nu_{R}^{j}=\left(\overline{3}, 1,-\frac{2}{3}\right), \\
\overline{Q_{L}^{i}} \vec{\tau} \gamma^{\mu} L_{L}^{j}=\left(\overline{3}, 3,-\frac{2}{3}\right), & \overline{u_{R}^{i}} \gamma^{\mu} e_{R}^{j}=\left(\overline{3}, 1,-\frac{5}{3}\right), & \overline{d_{R}^{i}} \gamma^{\mu} \nu_{R}^{j}=\left(\overline{3}, 1,+\frac{1}{3}\right),
\end{array}
$$

and their hermitian conjugates. Again, the bilinears in the first row share the same quantum numbers. The $F=2$ vectors are

$$
\begin{array}{ll}
\overline{d_{R}^{C i}} \gamma^{\mu} L_{L}^{j}=\left(3,2,-\frac{5}{6}\right), & \overline{u_{R}^{C i}} \gamma^{\mu} L_{L}^{j}=\left(3,2,+\frac{1}{6}\right), \\
\overline{Q_{L}^{C i}} \gamma^{\mu} e_{R}^{j}=\left(3,2,-\frac{5}{6}\right), & \overline{Q_{L}^{C i}} \gamma^{\mu} \nu_{R}^{j}=\left(3,2,+\frac{1}{6}\right),
\end{array}
$$

and their hermitian conjugates, the bilinears in the same columns sharing the same quantum numbers.

Introducing one scalar or vector leptoquark for each possible combination of quantum numbers, the most general Lagrangian which couple leptoquarks to these bilinears can be written down as

$$
\mathcal{L}=\mathcal{L}_{F=2}+\mathcal{L}_{F=0}
$$

where

$$
\begin{aligned}
\mathcal{L}_{F=2}= & {\left[y_{1 i j}^{L L}\left(\overline{Q_{L}^{C i}} \epsilon L_{L}^{j}\right)+y_{1 i j}^{R R}\left(\overline{u_{R}^{C i}} e_{R}^{j}\right)+y_{1 i j}^{\overline{R R}}\left(\overline{d_{R}^{C i}} \nu_{R}^{j}\right)\right] S_{1} } \\
& +\widetilde{y}_{1 i j}^{R R}\left(\overline{d_{R}^{C i}} e_{R}^{j}\right) \widetilde{S}_{1}+\bar{y}_{1 i j}^{R R}\left(\overline{u_{R}^{C i}} \nu_{R}^{j}\right) \bar{S}_{1}+y_{3 i j}^{L L}\left(\overline{Q_{L}^{C i}} \epsilon \vec{\tau} L_{L}^{j}\right) \vec{S}_{3} \\
& +\left[x_{2 i j}^{R L}\left(\overline{d_{R}^{C i}} \gamma^{\mu} L_{L}^{j}\right)+x_{2 i j}^{L R}\left(\overline{Q_{L}^{C i}} \gamma^{\mu} e_{R}^{j}\right)\right] \epsilon V_{2 \mu} \\
& +\left[\widetilde{x}_{2 i j}^{R L}\left(\overline{u_{R}^{C i}} \gamma^{\mu} L_{L}^{j}\right)+\widetilde{x}_{2 i j}^{L R}\left(\overline{Q_{L}^{C i}} \gamma^{\mu} \nu_{R}^{j}\right)\right] \epsilon \widetilde{V}_{2 \mu}+\text { h.c. }, \\
\mathcal{L}_{F=0}= & {\left[y_{2 i j}^{R L}\left(\overline{u_{R}^{i}} L_{L}^{j}\right) \epsilon+y_{2 i j}^{L R}\left(\overline{Q_{L}^{i}} e_{R}^{j}\right)\right] R_{2} } \\
& +\left[\widetilde{y}_{2 i j}^{R L}\left(\overline{d_{R}^{i}} L_{L}^{j}\right) \epsilon+\widetilde{y}_{2 i j}^{L R}\left(\overline{Q_{L}^{i}} \nu_{R}^{j}\right)\right] \widetilde{R}_{2} \\
& +\left[x_{1 i j}^{L L}\left(\overline{Q_{L}^{i}} \gamma^{\mu} L_{L}^{j}\right)+x_{1 i j}^{R R}\left(\overline{d_{R}^{i}} \gamma^{\mu} e_{R}^{j}\right)+x_{1 i j}^{\overline{R R}}\left(\overline{u_{R}^{i}} \gamma^{\mu} \nu_{R}^{j}\right)\right] U_{1 \mu} \\
& +\widetilde{x}_{1 i j}^{R R}\left(\overline{u_{R}^{i}} \gamma^{\mu} e_{R}^{j}\right) \widetilde{U}_{1 \mu}+\bar{x}_{1 i j}^{\overline{R R}}\left(\overline{d_{R}^{i}} \gamma^{\mu} \nu_{R}^{j}\right) \bar{U}_{1 \mu}+x_{3 i j}^{L L}\left(\overline{Q_{L}^{i}} \vec{\tau} \gamma^{\mu} L_{L}^{j}\right) \vec{U}_{3 \mu}+\text { h.c. . }
\end{aligned}
$$




\begin{tabular}{|c||c|c|c|c|c|}
\hline leptoquark & spin & $F=3 B+L$ & Quantum Numbers & $Q_{e m}=I_{3}^{L}+Y$ & Couples to \\
\hline \hline$S_{1}$ & 0 & -2 & $\left(\overline{3}, 1, \frac{1}{3}\right)$ & $\frac{1}{3}$ & $L L, R R$ \\
\hline$\widetilde{S}_{1}$ & 0 & -2 & $\left(\overline{3}, 1, \frac{4}{3}\right)$ & $\frac{4}{3}$ & $R R$ \\
\hline $\bar{S}_{1}$ & 0 & -2 & $\left(\overline{3}, 1,-\frac{2}{3}\right)$ & $-\frac{2}{3}$ & $\overline{R R}$ \\
\hline$S_{3}$ & 0 & -2 & $\left(\overline{3}, 3, \frac{1}{3}\right)$ & $\left(\frac{4}{3}, \frac{1}{3},-\frac{2}{3}\right)$ & $L L$ \\
\hline$R_{2}$ & 0 & 0 & $\left(3,2, \frac{7}{6}\right)$ & $\left(\frac{5}{3}, \frac{2}{3}\right)$ & $R L, L R$ \\
\hline$\widetilde{R}_{2}$ & 0 & 0 & $\left(3,2, \frac{1}{6}\right)$ & $\left(\frac{2}{3},-\frac{1}{3}\right)$ & $R L, \overline{L R}$ \\
\hline \hline$U_{1}$ & 1 & 0 & $\left(3,1, \frac{2}{3}\right)$ & $\frac{2}{3}$ & $L L, R R$ \\
\hline$\widetilde{U}_{1}$ & 1 & 0 & $\left(3,1, \frac{5}{3}\right)$ & $\frac{5}{3}$ & $R R$ \\
\hline $\bar{U}_{1}$ & 1 & 0 & $\left(3,1,-\frac{1}{3}\right)$ & $-\frac{1}{3}$ & $\overline{R R}$ \\
\hline$U_{3}$ & 1 & 0 & $\left(3,3, \frac{2}{3}\right)$ & $\left(\frac{5}{3}, \frac{2}{3},-\frac{1}{3}\right)$ & $L L$ \\
\hline$V_{2}$ & 1 & -2 & $\left(3,2, \frac{5}{6}\right)$ & $\left(\frac{4}{3}, \frac{1}{3}\right)$ & $R L, L R$ \\
\hline$\widetilde{V}_{2}$ & 1 & -2 & $\left(3,2,-\frac{1}{6}\right)$ & $\left(\frac{1}{3},-\frac{2}{3}\right)$ & $R L, \overline{L R}$ \\
\hline
\end{tabular}

Table 7. Quantum numbers of scalar and vector leptoquarks with $\mathrm{SU}(3)_{C} \times \mathrm{SU}(2)_{L} \times \mathrm{U}(1)_{Y}$ invariant couplings to quark-lepton pairs. $F=3 B+L$ is the fermion number and the hypercharge $Y$ is normalized so that $Q_{\mathrm{em}}=I_{3}^{L}+Y$. The final column indicates the chiralities of the quark and lepton fields that each leptoquark couples to, with the first letter of the pairs indicating the chirality of the quark field and the second letter indicating that of the lepton field. Pairs with a bar over them indicate that the right-handed lepton involved in the pair is a right-handed neutrino.

Here, the couplings between the vector/scalar leptoquarks and the fermions are denoted respectively by $x$ and $y$. The first subscript indicates the isospin of the leptoquark and the latter two the generations of the fermions involved. The superscripts denote the chiralities of the fermions, with a bar placed over the chiralities when a right-handed neutrino is involved. ${ }^{15}$

Thus, six scalar leptoquarks $S_{1}, \widetilde{S}_{1}, \bar{S}_{1}, S_{3}, R_{2}, \widetilde{R}_{2}$, and six vector leptoquarks $U_{1}, \widetilde{U}_{1}$, $\bar{U}_{1}, U_{3}, V_{2}, \widetilde{V}_{2}$ are introduced. Of these, $\bar{S}_{1}$ and $\bar{U}_{1}$ were absent in ref. [59] since the only lepton they couple to is the right-handed neutrino. The quantum numbers of these twelve fields are listed in table 7 . These leptoquarks can also have $\mathrm{SU}(3)_{C} \times \mathrm{SU}(2)_{L} \times \mathrm{U}(1)_{Y}$ invariant couplings to di-quarks as shown in ref. [58] but we will not discuss them here.

\footnotetext{
${ }^{15}$ In order to completely match the definitions of ref. [58], replace $x_{2 i j}^{R L}$ by $-x_{2 i j}^{R L}$, and $y_{2 i j}^{L R}$ by $y_{2 i j}^{L R *}$ (complex conjugate).
} 
Note that the subscripts of the leptoquark fields indicate their isospin. So $R_{2}, \widetilde{R}_{2}, V_{2}$, and $\widetilde{V}_{2}$ have two components each, while $S_{3}$ and $U_{3}$ have three components each. These can be written out as

$$
\begin{aligned}
& R_{2}=\left[\begin{array}{c}
R_{2}^{(5 / 3)} \\
R_{2}^{(2 / 3)}
\end{array}\right], \quad \tilde{R}_{2}=\left[\begin{array}{c}
\tilde{R}_{2}^{(2 / 3)} \\
\tilde{R}_{2}^{(-1 / 3)}
\end{array}\right], \quad S_{3}=\left[\begin{array}{c}
S_{3}^{(4 / 3)} \\
S_{3}^{(1 / 3)} \\
S_{3}^{(-2 / 3)}
\end{array}\right], \\
& V_{2}=\left[\begin{array}{c}
V_{2}^{(4 / 3)} \\
V_{2}^{(1 / 3)}
\end{array}\right], \quad \tilde{V}_{2}=\left[\begin{array}{c}
\tilde{V}_{2}^{(1 / 3)} \\
\tilde{V}_{2}^{(-2 / 3)}
\end{array}\right], \quad U_{3}=\left[\begin{array}{c}
U_{3}^{(5 / 3)} \\
U_{3}^{(2 / 3)} \\
U_{3}^{(-1 / 3)}
\end{array}\right] \text {, }
\end{aligned}
$$

where the superscripts in parentheses indicate the electromagnetic charge. Expanding out eqs. (A.7) and (A.8) in terms of these component fields, we find

$$
\begin{aligned}
\mathcal{L}_{F=2}= & {\left[y_{1 i j}^{L L}\left(\overline{u_{L}^{C i}} e_{L}^{j}-\overline{d_{L}^{C i}} \nu_{L}^{j}\right)+y_{1 i j}^{R R}\left(\overline{u_{R}^{C i}} e_{R}^{j}\right)+y_{1 i j}^{R R}\left(\overline{d_{R}^{C i}} \nu_{R}^{j}\right)\right] S_{1}^{(1 / 3)} } \\
+ & \widetilde{y}_{1 i j}^{R R}\left(\overline{d_{R}^{C i}} e_{R}^{j}\right) \widetilde{S}_{1}^{(4 / 3)}+\overline{y_{1 i j}^{R R}}\left(\overline{u_{R}^{C i}} \nu_{R}^{j}\right) \bar{S}_{1}^{(-2 / 3)} \\
+ & y_{3 i j}^{L L}\left[-\sqrt{2}\left(\overline{d_{L}^{C i}} e_{L}^{j}\right) S_{3}^{(4 / 3)}-\left(\overline{u_{L}^{C i}} e_{L}^{j}+\overline{d_{L}^{C i}} \nu_{L}^{j}\right) S_{3}^{(1 / 3)}+\sqrt{2}\left(\overline{u_{L}^{C i}} \nu_{L}^{j}\right) S_{3}^{(-2 / 3)}\right] \\
& -\left[x_{2 i j}^{R L}\left(\overline{d_{R}^{C i}} \gamma^{\mu} e_{L}^{j}\right)+x_{2 i j}^{L R}\left(\overline{d_{L}^{C i}} \gamma^{\mu} e_{R}^{j}\right)\right] V_{2 \mu}^{(4 / 3)}+\left[x_{2 i j}^{R L}\left(\overline{d_{R}^{C i}} \gamma^{\mu} \nu_{L}^{j}\right)+x_{2 i j}^{L R}\left(\overline{u_{L}^{C i}} \gamma^{\mu} e_{R}^{j}\right)\right] V_{2 \mu}^{(1 / 3)} \\
- & {\left[\widetilde{x}_{2 i j}^{R L}\left(\overline{u_{R}^{C i}} \gamma^{\mu} e_{L}^{j}\right)+\widetilde{x}_{2 i j}^{\overline{L R}}\left(\overline{d_{L}^{C i}} \gamma^{\mu} \nu_{R}^{j}\right)\right] \widetilde{V}_{2 \mu}^{(1 / 3)}+\left[\widetilde{x}_{2 i j}^{R L}\left(\overline{u_{R}^{C i}} \gamma^{\mu} \nu_{L}^{j}\right)+\widetilde{x}_{2 i j}^{\overline{L R}}\left(\overline{u_{L}^{C i}} \gamma^{\mu} \nu_{R}^{j}\right)\right] \widetilde{V}_{2 \mu}^{(-2 / 3)} } \\
+ & \text { h.c. }, \\
\mathcal{L}_{F=0}= & \left.-y_{2 i j}^{R L}\left(\overline{u_{R}^{i}} e_{L}^{j}\right)+y_{2 i j}^{L R}\left(\overline{u_{L}^{i}} e_{R}^{j}\right)\right] R_{2}^{(5 / 3)}+\left[y_{2 i j}^{R L}\left(\overline{u_{R}^{i}} \nu_{L}^{j}\right)+y_{2 i j}^{L R}\left(\overline{d_{L}^{i}} e_{R}^{j}\right)\right] R_{2}^{(2 / 3)} \\
& +\left[-\widetilde{y}_{2 i j}^{R L}\left(\overline{d_{R}^{i}} e_{L}^{j}\right)+\widetilde{y}_{2 i j}^{\overline{L R}}\left(\overline{u_{L}^{i}} \nu_{R}^{j}\right)\right] \widetilde{R}_{2}^{(2 / 3)}+\left[\widetilde{y}_{2 i j}^{R L}\left(\overline{d_{R}^{i}} \nu_{L}^{j}\right)+\widetilde{y}_{2 i j}^{\overline{L R}}\left(\overline{d_{L}^{i}} \nu_{R}^{j}\right)\right] \widetilde{R}_{2}^{(-1 / 3)} \\
& +\left[x_{1 i j}^{L L}\left(\overline{u_{L}^{i}} \gamma^{\mu} \nu_{L}^{j}+\overline{d_{L}^{i}} \gamma^{\mu} e_{L}^{j}\right)+x_{1 i j}^{R R}\left(\overline{d_{R}^{i}} \gamma^{\mu} e_{R}^{j}\right)+x_{1 i j}^{\overline{R R}}\left(\overline{u_{R}^{i}} \gamma^{\mu} \nu_{R}^{j}\right)\right] U_{1 \mu}^{(2 / 3)} \\
& +\widetilde{x}_{1 i j}^{R R}\left(\overline{u_{R}^{i}} \gamma^{\mu} e_{R}^{j}\right) \widetilde{U}_{1 \mu}^{(5 / 3)}+\bar{x}_{1 i j}^{\overline{R R}}\left(\overline{d_{R}^{i}} \gamma^{\mu} \nu_{R}^{j}\right) \overline{U_{1 \mu}^{(-1 / 3)}} \\
& +x_{3 i j}^{L L}\left[\sqrt{2}\left(\overline{u_{L}^{i}} \gamma^{\mu} e_{L}^{j}\right) U_{3 \mu}^{(5 / 3)}+\left(\overline{u_{L}^{i}} \gamma^{\mu} \nu_{L}^{j}-\overline{d_{L}^{i}} \gamma^{\mu} e_{L}^{j}\right) U_{3 \mu}^{(2 / 3)}+\sqrt{2}\left(\overline{d_{L}^{i}} \gamma^{\mu} \nu_{L}^{j}\right) U_{3 \mu}^{(-1 / 3)}\right] \\
& + \text { h.c. . } \\
& \text { A.11) }
\end{aligned}
$$

where we have added superscripts indicating the electromagnetic charges to the isosinglet fields also.

Open Access. This article is distributed under the terms of the Creative Commons Attribution License (CC-BY 4.0), which permits any use, distribution and reproduction in any medium, provided the original author(s) and source are credited. 


\section{References}

[1] U. Aydemir, D. Minic, C. Sun and T. Takeuchi, Pati-Salam unification from noncommutative geometry and the TeV-scale $W_{R}$ boson, Int. J. Mod. Phys. A 31 (2016) 1550223 [arXiv:1509.01606] [INSPIRE].

[2] U. Aydemir, D. Minic, C. Sun and T. Takeuchi, The 750 GeV diphoton excess in unified $\mathrm{SU}(2)_{L} \times \mathrm{SU}(2)_{R} \times \mathrm{SU}(4)$ models from noncommutative geometry, Mod. Phys. Lett. A 31 (2016) 1650101 [arXiv:1603.01756] [INSPIRE].

[3] A.H. Chamseddine, A. Connes and W.D. van Suijlekom, Beyond the Spectral Standard Model: Emergence of Pati-Salam Unification, JHEP 11 (2013) 132 [arXiv:1304.8050] [INSPIRE].

[4] A.H. Chamseddine, A. Connes and W.D. van Suijlekom, Grand Unification in the Spectral Pati-Salam Model, JHEP 11 (2015) 011 [arXiv:1507.08161] [INSPIRE].

[5] A. Connes, Noncommutative geometry, Academic Press, New York U.S.A. (1994).

[6] A. Connes and M. Marcolli, Noncommutative Geometry, Quantum Fields and Motives, American Mathematical Society, Providence U.S.A. (2007).

[7] A.H. Chamseddine and A. Connes, Noncommutative Geometry as a Framework for Unification of all Fundamental Interactions including Gravity. Part I, Fortsch. Phys. 58 (2010) 553 [arXiv: 1004.0464] [INSPIRE].

[8] A.H. Chamseddine and A. Connes, Resilience of the Spectral Standard Model, JHEP 09 (2012) 104 [arXiv:1208.1030] [INSPIRE].

[9] R.N. Mohapatra, Unification and Supersymmetry - The Frontiers of Quark-Lepton Physics, third edition, Springer, Berlin Germany (2002).

[10] J.C. Pati and A. Salam, Lepton Number as the Fourth Color, Phys. Rev. D 10 (1974) 275 [Erratum ibid. D 11 (1975) 703] [INSPIRE].

[11] R.N. Mohapatra and J.C. Pati, A Natural Left-Right Symmetry, Phys. Rev. D 11 (1975) 2558 [INSPIRE].

[12] R.N. Mohapatra and J.C. Pati, Left-Right Gauge Symmetry and an Isoconjugate Model of CP-violation, Phys. Rev. D 11 (1975) 566 [InSPIRE].

[13] G. Senjanović and R.N. Mohapatra, Exact Left-Right Symmetry and Spontaneous Violation of Parity, Phys. Rev. D 12 (1975) 1502 [InSPIRE].

[14] ATLAS collaboration, Search for new phenomena in the dijet mass distribution using $p-p$ collision data at $\sqrt{s}=8 \mathrm{TeV}$ with the ATLAS detector, Phys. Rev. D 91 (2015) 052007 [arXiv: 1407.1376] [INSPIRE].

[15] ATLAS collaboration, Search for high-mass diboson resonances with boson-tagged jets in proton-proton collisions at $\sqrt{s}=8 \mathrm{TeV}$ with the ATLAS detector, JHEP 12 (2015) 055 [arXiv: 1506.00962] [INSPIRE].

[16] ATLAS collaboration, Combination of searches for $W W, W Z$ and $Z Z$ resonances in $p p$ collisions at $\sqrt{s}=8 \mathrm{TeV}$ with the ATLAS detector, Phys. Lett. B 755 (2016) 285 [arXiv: 1512.05099] [INSPIRE].

[17] CMS collaboration, Search for massive resonances decaying into pairs of boosted bosons in semi-leptonic final states at $\sqrt{s}=8 \mathrm{TeV}$, JHEP 08 (2014) 174 [arXiv:1405.3447] [INSPIRE]. 
[18] CMS collaboration, Search for resonances and quantum black holes using dijet mass spectra in proton-proton collisions at $\sqrt{s}=8 \mathrm{TeV}$, Phys. Rev. D 91 (2015) 052009 [arXiv: 1501.04198] [INSPIRE].

[19] CMS collaboration, Search for massive WH resonances decaying into the $\ell \nu \mathrm{b} \overline{\mathrm{b}}$ final state at $\sqrt{s}=8 \mathrm{TeV}$, Eur. Phys. J. C 76 (2016) 237 [arXiv:1601.06431] [INSPIRE].

[20] CMS collaboration, Search for new physics in high mass diphoton events in proton-proton collisions at 13 TeV, CMS-PAS-EXO-15-004 (2015).

[21] ATLAS collaboration, Search for resonances decaying to photon pairs in 3.2 $\mathrm{fb}^{-1}$ of $\mathrm{pp}$ collisions at $\sqrt{s}=13$ TeV with the ATLAS detector, ATLAS-CONF-2015-081 (2015).

[22] CMS collaboration, Search for a heavy right-handed $W$ boson and a heavy neutrino in events with two same-flavor leptons and two jets at $\sqrt{s}=13$ TeV, JHEP 05 (2018) 148 [arXiv: 1803.11116] [INSPIRE].

[23] ATLAS collaboration, Search for new phenomena in high-mass diphoton final states using $37 \mathrm{fb}^{-1}$ of proton-proton collisions collected at $\sqrt{s}=13$ TeV with the ATLAS detector, Phys. Lett. B 775 (2017) 105 [arXiv:1707.04147] [INSPIRE].

[24] ATLAS collaboration, Search for new phenomena in high-mass diphoton final states with ATLAS at $\sqrt{s}=13$ TeV, PoS(EPS-HEP2017) 710 .

[25] G. Ciezarek et al., A Challenge to Lepton Universality in B Meson Decays, Nature 546 (2017) 227 [arXiv: 1703.01766] [INSPIRE].

[26] M. Bauer and M. Neubert, Minimal Leptoquark Explanation for the $R_{D^{(*)}}, R_{K}$ and $(g-2)_{g}$ Anomalies, Phys. Rev. Lett. 116 (2016) 141802 [arXiv:1511.01900] [INSPIRE].

[27] D. Bečirević, N. Košnik, O. Sumensari and R. Zukanovich Funchal, Palatable Leptoquark Scenarios for Lepton Flavor Violation in Exclusive $b \rightarrow s \ell_{1} \ell_{2}$ modes, JHEP 11 (2016) 035 [arXiv: 1608.07583] [INSPIRE].

[28] Y. Cai, J. Gargalionis, M.A. Schmidt and R.R. Volkas, Reconsidering the One Leptoquark solution: flavor anomalies and neutrino mass, JHEP 10 (2017) 047 [arXiv:1704.05849] [INSPIRE].

[29] M. Freytsis, Z. Ligeti and J.T. Ruderman, Flavor models for $\bar{B} \rightarrow D^{(*)} \tau \bar{\nu}$, Phys. Rev. D 92 (2015) 054018 [arXiv:1506.08896] [INSPIRE].

[30] A. Crivellin, D. Müller and T. Ota, Simultaneous explanation of $R\left(D^{()}\right)$and $b \rightarrow s \mu^{+} \mu^{-}:$the last scalar leptoquarks standing, JHEP 09 (2017) 040 [arXiv:1703.09226] [INSPIRE].

[31] D. Marzocca, Addressing the B-physics anomalies in a fundamental Composite Higgs Model, JHEP 07 (2018) 121 [arXiv: 1803.10972] [INSPIRE].

[32] D. Buttazzo, A. Greljo, G. Isidori and D. Marzocca, B-physics anomalies: a guide to combined explanations, JHEP 11 (2017) 044 [arXiv: 1706.07808] [INSPIRE].

[33] H. Murayama and T. Yanagida, A viable SU(5) GUT with light leptoquark bosons, Mod. Phys. Lett. A 7 (1992) 147 [INSPIRE].

[34] P. Cox, A. Kusenko, O. Sumensari and T.T. Yanagida, SU(5) Unification with TeV-scale Leptoquarks, JHEP 03 (2017) 035 [arXiv:1612.03923] [INSPIRE].

[35] LHCb collaboration, Test of lepton universality using $B^{+} \rightarrow K^{+} \ell^{+} \ell^{-}$decays, Phys. Rev. Lett. 113 (2014) 151601 [arXiv:1406.6482] [INSPIRE]. 
[36] LHCb collaboration, Test of lepton universality with $B^{0} \rightarrow K^{* 0} \ell^{+} \ell^{-}$decays, JHEP 08 (2017) 055 [arXiv : 1705.05802] [INSPIRE].

[37] S. Bifani, Search for New Physics with $b \rightarrow$ sll decays at LHCb, https://indico.cern.ch/event/580620/attachments/1442409/2226501/cern_2017_04_18.pdf (2017).

[38] LHCb collaboration, Measurement of the ratio of branching fractions $\mathcal{B}\left(\bar{B}^{0} \rightarrow D^{*+} \tau^{-} \bar{\nu}_{\tau}\right) / \mathcal{B}\left(\bar{B}^{0} \rightarrow D^{*+} \mu^{-} \bar{\nu}_{\mu}\right)$, Phys. Rev. Lett. 115 (2015) 111803 [Erratum ibid. 115 (2015) 159901] [arXiv: 1506.08614] [INSPIRE].

[39] G. Wormser, $R_{D^{*}}$ status: overview and prospects, https://indico.cern.ch/event/586719/ contributions/2531261/attachments/1470695/2275578/2_fpcp_talk_wormser.pdf.

[40] Belle collaboration, M. Huschle et al., Measurement of the branching ratio of $\bar{B} \rightarrow D^{(*)} \tau^{-} \bar{\nu}_{\tau}$ relative to $\bar{B} \rightarrow D^{(*)} \ell^{-} \bar{\nu}_{\ell}$ decays with hadronic tagging at Belle, Phys. Rev. D 92 (2015) 072014 [arXiv: 1507.03233] [INSPIRE].

[41] Belle collaboration, Y. Sato et al., Measurement of the branching ratio of $\bar{B}^{0} \rightarrow D^{*+} \tau^{-} \bar{\nu}_{\tau}$ relative to $\bar{B}^{0} \rightarrow D^{*+} \ell^{-} \bar{\nu}_{\ell}$ decays with a semileptonic tagging method, Phys. Rev. D 94 (2016) 072007 [arXiv: 1607.07923] [INSPIRE].

[42] Belle collaboration, S. Hirose et al., Measurement of the $\tau$ lepton polarization and $R\left(D^{*}\right)$ in the decay $\bar{B} \rightarrow D^{*} \tau^{-} \bar{\nu}_{\tau}$, Phys. Rev. Lett. 118 (2017) 211801 [arXiv:1612. 00529] [INSPIRE].

[43] BaBar collaboration, J.P. Lees et al., Evidence for an excess of $\bar{B} \rightarrow D^{(*)} \tau^{-} \bar{\nu}_{\tau}$ decays, Phys. Rev. Lett. 109 (2012) 101802 [arXiv: 1205.5442] [INSPIRE].

[44] BaBar collaboration, J.P. Lees et al., Measurement of an Excess of $\bar{B} \rightarrow D^{(*)} \tau^{-} \bar{\nu}_{\tau}$ Decays and Implications for Charged Higgs Bosons, Phys. Rev. D 88 (2013) 072012 [arXiv: 1303.0571] [INSPIRE].

[45] G. Hiller and F. Krüger, More model-independent analysis of $b \rightarrow s$ processes, Phys. Rev. D 69 (2004) 074020 [hep-ph/0310219] [INSPIRE].

[46] C. Bobeth, G. Hiller and G. Piranishvili, Angular distributions of $\bar{B} \rightarrow \bar{K} \ell^{+} \ell^{-}$decays, JHEP 12 (2007) 040 [arXiv:0709.4174] [INSPIRE].

[47] HPQCD collaboration, C. Bouchard, G.P. Lepage, C. Monahan, H. Na and J. Shigemitsu, Standard Model Predictions for $B \rightarrow K \ell^{+} \ell^{-}$with Form Factors from Lattice QCD, Phys. Rev. Lett. 111 (2013) 162002 [Erratum ibid. 112 (2014) 149902] [arXiv:1306. 0434] [INSPIRE].

[48] G. D'Amico et al., Flavour anomalies after the $R_{K^{*}}$ measurement, JHEP 09 (2017) 010 [arXiv: 1704.05438] [INSPIRE].

[49] A.K. Alok, B. Bhattacharya, A. Datta, D. Kumar, J. Kumar and D. London, New Physics in $b \rightarrow s \mu^{+} \mu^{-}$after the Measurement of $R_{K^{*}}$, Phys. Rev. D 96 (2017) 095009 [arXiv: 1704.07397] [INSPIRE].

[50] M. Bordone, G. Isidori and A. Pattori, On the Standard Model predictions for $R_{K}$ and $R_{K^{*}}$, Eur. Phys. J. C 76 (2016) 440 [arXiv:1605. 07633] [InSPIRE].

[51] B. Capdevila, S. Descotes-Genon, L. Hofer and J. Matias, Hadronic uncertainties in $B \rightarrow K^{*} \mu^{+} \mu^{-}$: a state-of-the-art analysis, JHEP 04 (2017) 016 [arXiv:1701.08672] [INSPIRE]. 
[52] Heavy Flavor Averaging Group, Average of $R_{D}$ and $R_{D *}$ for FPCP 2017, http://www.slac.stanford.edu/xorg/hfag/semi/fpcp17/RDRDs.html (2017).

[53] S. Fajfer, J.F. Kamenik and I. Nisandzic, On the $B \rightarrow D^{*} \tau \bar{\nu}_{\tau}$ Sensitivity to New Physics, Phys. Rev. D 85 (2012) 094025 [arXiv:1203.2654] [INSPIRE].

[54] S. Aoki et al., Review of lattice results concerning low-energy particle physics, Eur. Phys. J. C 77 (2017) 112 [arXiv:1607.00299] [INSPIRE].

[55] S. Jaiswal, S. Nandi and S.K. Patra, Extraction of $\left|V_{c b}\right|$ from $B \rightarrow D^{(*)} \ell \nu_{\ell}$ and the Standard Model predictions of $R\left(D^{(*)}\right)$, JHEP 12 (2017) 060 [arXiv:1707.09977] [INSPIRE].

[56] D. Bigi, P. Gambino and S. Schacht, $R\left(D^{*}\right),\left|V_{c b}\right|$ and the Heavy Quark Symmetry relations between form factors, JHEP 11 (2017) 061 [arXiv:1707.09509] [INSPIRE].

[57] S.L. Glashow, D. Guadagnoli and K. Lane, Lepton Flavor Violation in B Decays?, Phys. Rev. Lett. 114 (2015) 091801 [arXiv:1411.0565] [INSPIRE].

[58] I. Doršner, S. Fajfer, A. Greljo, J.F. Kamenik and N. Košnik, Physics of leptoquarks in precision experiments and at particle colliders, Phys. Rept. 641 (2016) 1 [arXiv:1603.04993] [INSPIRE].

[59] W. Buchmüller, R. Rückl and D. Wyler, Leptoquarks in Lepton - Quark Collisions, Phys. Lett. B 191 (1987) 442 [Erratum ibid. B 448 (1999) 320] [INSPIRE].

[60] B. Grinstein, M.J. Savage and M.B. Wise, $B \rightarrow X(s) e^{+} e^{-}$in the Six Quark Model, Nucl. Phys. B 319 (1989) 271 [INSPIRE].

[61] M. Misiak, The $b \rightarrow s e^{+} e^{-}$and $b \rightarrow s \gamma$ decays with next-to-leading logarithmic $Q C D$ corrections, Nucl. Phys. B 393 (1993) 23 [Erratum ibid. B 439 (1995) 461] [INSPIRE].

[62] A.J. Buras and M. Münz, Effective Hamiltonian for $B \rightarrow X(s) e^{+} e^{-}$beyond leading logarithms in the NDR and HV schemes, Phys. Rev. D 52 (1995) 186 [hep-ph/9501281] [INSPIRE].

[63] G. Hiller and M. Schmaltz, $R_{K}$ and future $b \rightarrow$ sll physics beyond the standard model opportunities, Phys. Rev. D 90 (2014) 054014 [arXiv: 1408.1627] [INSPIRE].

[64] D. Aloni, A. Dery, C. Frugiuele and Y. Nir, Testing minimal flavor violation in leptoquark models of the $R_{K^{(*)}}$ anomaly, JHEP 11 (2017) 109 [arXiv:1708.06161] [INSPIRE].

[65] D. Bečirević and O. Sumensari, A leptoquark model to accommodate $R_{K}^{\exp }<R_{K}^{\mathrm{SM}}$ and $R_{K^{*}}^{\exp }<R_{K^{*}}^{\mathrm{SM}}$, JHEP 08 (2017) 104 [arXiv: 1704.05835] [INSPIRE].

[66] D. Bečirević, S. Fajfer and N. Košnik, Lepton flavor nonuniversality in $b \rightarrow s \ell^{+} \ell^{-}$processes, Phys. Rev. D 92 (2015) 014016 [arXiv: 1503.09024] [INSPIRE].

[67] D. Bečirević, S. Fajfer, N. Košnik and O. Sumensari, Leptoquark model to explain the B-physics anomalies, $R_{K}$ and $R_{D}$, Phys. Rev. D 94 (2016) 115021 [arXiv:1608.08501] [INSPIRE].

[68] F.S. Queiroz, K. Sinha and A. Strumia, Leptoquarks, Dark Matter and Anomalous LHC Events, Phys. Rev. D 91 (2015) 035006 [arXiv:1409.6301] [INSPIRE].

[69] S. Descotes-Genon, L. Hofer, J. Matias and J. Virto, Global analysis of $b \rightarrow$ sll anomalies, JHEP 06 (2016) 092 [arXiv:1510.04239] [INSPIRE].

[70] F. Mahmoudi, T. Hurth and S. Neshatpour, Present Status of $b \rightarrow s \ell^{+} \ell^{-}$Anomalies, Nucl. Part. Phys. Proc. 285-286 (2017) 39 [arXiv:1611.05060] [INSPIRE]. 
[71] W. Altmannshofer, C. Niehoff, P. Stangl and D.M. Straub, Status of the $B \rightarrow K^{*} \mu^{+} \mu^{-}$ anomaly after Moriond 2017, Eur. Phys. J. C 77 (2017) 377 [arXiv:1703.09189] [INSPIRE].

[72] A.H. Chamseddine and A. Connes, Why the Standard Model, J. Geom. Phys. 58 (2008) 38 [arXiv:0706.3688] [INSPIRE].

[73] U. Aydemir and T. Mandal, LHC probes of TeV-scale scalars in $\mathrm{SO}(10)$ grand unification, Adv. High Energy Phys. 2017 (2017) 7498795 [arXiv:1601.06761] [INSPIRE].

[74] M.A. Kurkov and F. Lizzi, Clifford Structures in Noncommutative Geometry and the Extended Scalar Sector, Phys. Rev. D 97 (2018) 085024 [arXiv:1801.00260] [INSPIRE].

[75] T. Takeuchi, Special relativity, in Encyclopedia of applied high energy and particle physics, R. Stock eds., Wiley-VCH, New York U.S.A. (2009), pg. 47.

[76] U. Aydemir, D. Minic, C. Sun and T. Takeuchi, Higgs mass, superconnections and the TeV-scale left-right symmetric model, Phys. Rev. D 91 (2015) 045020 [arXiv:1409.7574] [INSPIRE].

[77] G. Altarelli and D. Meloni, A non supersymmetric $\mathrm{SO}(10)$ grand unified model for all the physics below $M_{G U T}$, JHEP 08 (2013) 021 [arXiv:1305.1001] [INSPIRE].

[78] D.R.T. Jones, The Two Loop $\beta$-function for a $G_{1} \times G_{2}$ Gauge Theory, Phys. Rev. D 25 (1982) 581 [INSPIRE].

[79] M. Lindner and M. Weiser, Gauge coupling unification in left-right symmetric models, Phys. Lett. B 383 (1996) 405 [hep-ph/9605353] [INSPIRE].

[80] Particle Data Group collaboration, C. Patrignani et al., Review of Particle Physics, Chin. Phys. C 40 (2016) 100001 [inSPIRE].

[81] SLD Electroweak Group, DELPHI, ALEPH, SLD, SLD Heavy Flavour Group, OPAL, LEP Electroweak Working Group and L3 collaborations, S. Schael et al., Precision electroweak measurements on the $Z$ resonance, Phys. Rept. 427 (2006) 257 [hep-ex/0509008] [INSPIRE].

[82] R. Slansky, Group Theory for Unified Model Building, Phys. Rept. 79 (1981) 1 [INSPIRE].

[83] Super-Kamiokande collaboration, V. Takhistov, Review of Nucleon Decay Searches at Super-Kamiokande, in Proceedings of 51st Rencontres de Moriond on Electroweak Interactions and Unified Theories, La Thuile Italy (2016), pg. 437 [arXiv:1605.03235] [INSPIRE].

[84] U. Aydemir, $\mathrm{SO}(10)$ grand unification in light of recent LHC searches and colored scalars at the TeV-scale, Int. J. Mod. Phys. A 31 (2016) 1650034 [arXiv:1512.00568] [INSPIRE].

[85] R.N. Mohapatra and R.E. Marshak, Local B-L Symmetry of Electroweak Interactions, Majorana Neutrinos and Neutron Oscillations, Phys. Rev. Lett. 44 (1980) 1316 [Erratum ibid. 44 (1980) 1643] [INSPIRE].

[86] A.H. Chamseddine and A. Connes, The Spectral action principle, Commun. Math. Phys. 186 (1997) 731 [hep-th/9606001] [inSPIRE].

[87] U. Aydemir, D. Minic and T. Takeuchi, The Higgs Mass and the Emergence of New Physics, Phys. Lett. B 724 (2013) 301 [arXiv:1304.6092] [InSPIRE].

[88] L. Freidel, R.G. Leigh and D. Minic, Noncommutativity of closed string zero modes, Phys. Rev. D 96 (2017) 066003 [arXiv:1707.00312] [INSPIRE].

[89] L. Freidel, R.G. Leigh and D. Minic, Intrinsic non-commutativity of closed string theory, JHEP 09 (2017) 060 [arXiv:1706.03305] [INSPIRE]. 
[90] L. Freidel, R.G. Leigh and D. Minic, Modular Spacetime and Metastring Theory, J. Phys. Conf. Ser. 804 (2017) 012032 [INSPIRE].

[91] L. Freidel, R.G. Leigh and D. Minic, Quantum Spaces are Modular, Phys. Rev. D 94 (2016) 104052 [arXiv: 1606.01829] [INSPIRE].

[92] L. Freidel, R.G. Leigh and D. Minic, Metastring Theory and Modular Space-time, JHEP 06 (2015) 006 [arXiv: 1502.08005] [INSPIRE].

[93] L. Freidel, R.G. Leigh and D. Minic, Quantum Gravity, Dynamical Phase Space and String Theory, Int. J. Mod. Phys. D 23 (2014) 1442006 [arXiv:1405.3949] [INSPIRE].

[94] L. Freidel, R.G. Leigh and D. Minic, Born Reciprocity in String Theory and the Nature of Spacetime, Phys. Lett. B 730 (2014) 302 [arXiv:1307.7080] [INSPIRE]. 